\title{
ALGEBRO-GEOMETRIC FEYNMAN RULES
}

\author{
PAOLO ALUFFI AND MATILDE MARCOLLI
}

\begin{abstract}
We give a general procedure to construct algebro-geometric Feynman rules, that is, characters of the Connes-Kreimer Hopf algebra of Feynman graphs that factor through a Grothendieck ring of immersed conical varieties, via the class of the complement of the affine graph hypersurface. In particular, this maps to the usual Grothendieck ring of varieties, defining motivic Feynman rules. We also construct an algebro-geometric Feynman rule with values in a polynomial ring, which does not factor through the usual Grothendieck ring, and which is defined in terms of characteristic classes of singular varieties. This invariant recovers, as a special value, the Euler characteristic of the projective graph hypersurface complement. The main result underlying the construction of this invariant is a formula for the characteristic classes of the join of two projective varieties. We discuss the BPHZ renormalization procedure in this algebro-geometric context and some motivic zeta functions arising from the partition functions associated to motivic Feynman rules.
\end{abstract}

\section{INTRODUCTION}

In 3 we presented explicit computations of classes in the Grothendieck ring of varieties, of Chern-Schwartz-MacPherson characteristic classes, and by specialization Euler characteristics, for some particular classes of graph hypersurfaces. The latter are singular projective hypersurfaces associated to the parametric formulation of Feynman integrals in scalar quantum field theories and have recently been the object of extensive investigation (see [6], 7], 8], 9], 25], 26]).

The purpose of the present paper is to answer a question posed to us by the referee of [3. We describe the problem here briefly, along with the necessary background. All this will be discussed in more details in the body of the paper.

For us a Feynman graph $\Gamma$ will be a finite graph whose set of edges consists of internal edges $E_{\text {int }}(\Gamma)$ and external edges $E_{\text {ext }}(\Gamma)$. Whenever we focus on invariants that only involve internal edges, we can assume that $\Gamma$ is just a graph in the ordinary sense.

Consider a graph $\Gamma$ consisting of two components, $\Gamma=\Gamma_{1} \cup \Gamma_{2}$. To each component we can associate a corresponding graph hypersurface $X_{\Gamma_{1}} \subset \mathbb{P}^{n_{1}-1}$ and $X_{\Gamma_{2}} \subset \mathbb{P}^{n_{2}-1}$, where $n_{i}=\# E_{\text {int }}\left(\Gamma_{i}\right)$ is the number of internal edges of the Feynman graph $\Gamma_{i}$. The Feynman integral $U\left(\Gamma_{i}, p_{i}\right)$, with assigned external momenta $p_{i}=\left(p_{i}\right)_{e}$ for $e \in E_{\text {ext }}\left(\Gamma_{i}\right)$, is computed in the parametric form as an integral over a simplex $\sigma_{n_{i}}$ of an algebraic differential form defined on the hypersurface complement $\mathbb{P}^{n_{i}-1} \backslash X_{\Gamma_{i}}$. The multiplicative property of the Feynman rules implies that, for a graph $\Gamma=\Gamma_{1} \cup \Gamma_{2}$, one correspondingly has $U(\Gamma, p)=U\left(\Gamma_{1}, p_{1}\right) U\left(\Gamma_{2}, p_{2}\right)$, so that it is customary in quantum field theory to pass from the partition function whose asymptotic series involves all graphs to the one that only involves connected graphs. A further simplification of the combinatorics of graphs that takes place in quantum field theory is obtained by passing to the 1PI effective action, which only involves graphs that are 2-edge-connected (1-particle irreducible in the physics terminology), i.e. that cannot be disconnected by removal of a single edge.

The Connes-Kreimer theory [10, 11] (see also [12]) shows that the Feynman rules define a character of the Connes-Kreimer Hopf algebra $\mathcal{H}$ of Feynman graphs. Namely, the collection of dimensionally regularized Feynman integrals $U(\Gamma, p)$ of all the 1PI graphs 
of a given scalar quantum field theory defines a homomorphism of unital commutative algebras $\phi \in \operatorname{Hom}(\mathcal{H}, \mathcal{K})$, where $\mathcal{K}$ is the field of germs of meromorphic functions at $z=0 \in \mathbb{C}$. The coproduct in the Hopf algebra is then used in [10] to obtain a recursive formula for the Birkhoff factorization of loops in the pro-unipotent complex Lie group $G(\mathbb{C})=\operatorname{Hom}(\mathcal{H}, \mathbb{C})$. This provides the counterterms and the renormalized values of all the Feynman integrals in the form of what is known in physics as the Bogolyubov recursion, or BPHZ renormalization procedure.

In particular, any character of the Hopf algebra $\mathcal{H}$ can be thought of as a possible assignment of Feynman rules for the given field theory, and the renormalization procedure can be applied to any such character as to the case of the Feynman integrals. In turn, the characters need not necessarily take values in the field $\mathcal{K}$ of convergent Laurent series for the BPHZ renormalization procedure to make sense.

In fact, it was shown in [14 how the same Connes-Kreimer recursive formula for the Birkhoff factorization of loops continues to work unchanged whenever the target of the Hopf algebra character is a Rota-Baxter algebra of weight $\lambda=-1$. In the Connes-Kreimer case, it is the operator of projection of a Laurent series onto its divergent part that is a Rota-Baxter operator and the Rota-Baxter identity is what is needed to show that, in the Birkhoff factorization $\phi=\left(\phi_{-} \circ S\right) \star \phi_{+}$, with $S$ the antipode and $\star$ the product dual to the coproduct, the two terms $\phi_{ \pm}$are also algebra homomorphisms.

When working in the algebro-geometric world of the graph hypersurfaces $X_{\Gamma}$, one would like to have "motivic Feynman rules", namely an assignment of an Euler characteristic $\chi_{n e w}$ (the class in the Grothendieck ring of varieties is a universal Euler characteristic) to the graph hypersurface complements $\mathbb{P}^{n-1} \backslash X_{\Gamma}$ with the property that, in the case of graphs $\Gamma$ consisting of several disjoint components $\Gamma_{1} \ldots, \Gamma_{k}$, one has

$$
\chi_{\text {new }}\left(\mathbb{P}^{n-1} \backslash X_{\Gamma}\right)=\prod_{i} \chi_{n e w}\left(\mathbb{P}^{n_{i}-1} \backslash X_{\Gamma_{i}}\right),
$$

as in the case of the Feynman integrals $U(\Gamma, p)=\prod_{i} U\left(\Gamma_{i}, p_{i}\right)$. Here the graph hypersurface $X_{\Gamma}$ associated to a graph $\Gamma$ is defined as the hypersurface in $\mathbb{P}^{n-1}\left(n=\# E_{\text {int }}(\Gamma)\right)$ given by the vanishing of the polynomial

$$
\Psi_{\Gamma}\left(t_{1}, \ldots, t_{n}\right)=\sum_{T \subset \Gamma} \prod_{e \notin T} t_{e},
$$

with the sum over spanning forests $T$ of $\Gamma$, and the product of the edge variables $t_{e}$ of the edges $e$ of $\Gamma$ that are not in the forest $T$. If $\Gamma=\Gamma_{1} \cup \Gamma_{2}$ is a disjoint union, then clearly

$$
\Psi_{\Gamma}\left(t_{1}, \ldots, t_{n}\right)=\Psi_{\Gamma_{1}}\left(t_{1}, \ldots, t_{n_{1}}\right) \Psi_{\Gamma_{2}}\left(t_{n_{1}+1}, \ldots, t_{n_{1}+n_{2}}\right) .
$$

One can see that the usual Euler characteristic does not satisfy the desired property (1.1). In fact, if $\Gamma$ is not a forest, one can see that the hypersurface complement $\mathbb{P}^{n-1} \backslash X_{\Gamma}$ is a $\mathbb{G}_{m}$-bundle over the product $\left(\mathbb{P}^{n_{1}-1} \backslash X_{\Gamma_{1}}\right) \times\left(\mathbb{P}^{n_{2}-1} \backslash X_{\Gamma_{2}}\right)$, hence its Euler characteristic vanishes and the multiplicative property cannot be satisfied.

The question the referee of [3] asked us is whether there exists a natural modification $\chi_{\text {new }}$ of the usual Euler characteristic that restores the multiplicative property (1.1), thus giving an interesting example of algebro-geometric Feynman rules. The main result of the present paper is to show that indeed such modifications of the Euler characteristic exist and they can be obtained from already well known natural enhancements of the Euler characteristic in the context of algebraic geometry. In particular, we produce one such invariant obtained using classes in the Grothendieck ring of varieties, and one obtained using the Chern-Schwartz-MacPherson characteristic class of singular algebraic varieties, 24] 28, and we show that both descend from a common invariant that lives in a "Grothendieck ring of immersed conical varieties". 
The first case, where one considers an invariant with values in the usual Grothendieck ring, has the advantage that it is motivic, so it indeed defines "motivic Feynman rules" as the referee suggested, and also it is in general easier to compute explicitly, while the second case where the invariant is constructed in terms of characteristic classes is more difficult to compute, but it has the advantage that it takes values in a more manageable polynomial ring. We discuss the meaning of the BPHZ renormalization procedure in the Connes-Kreimer form for some of these invariants, using suitable Rota-Baxter operators on polynomial algebras.

The paper is structured as follows. We recall briefly in $\$ 1.1$ the properties of Feynman integrals and Feynman rules in perturbative scalar quantum field theory, as those serve as a model for our algebro-geometric definition. In $\$ 2$ we introduce the notion of algebrogeometric Feynman rules, by requiring that the multiplicative invariants associated to graphs depend on the data of the affine hypersurface complement, up to linear changes of coordinates. We show in $\$ 2.1$ that there is a universal algebro-geometric Feynman rule that takes values in a suitably defined Grothendieck ring of immersed conical varieties, $\mathcal{F}$. We show how the values behave under simple operations on graphs, such as bisecting and edge, connecting graphs by a vertex or an edge, etc. The existence of this universal algebrogeometric Feynman rule is based on the multiplicative property of the affine hypersurface complements over disjoint unions of graphs, which does not hold in the projective setting. We then show in 2.3 that the universal Feynman rule maps to a motivic Feynman rule with values in the usual Grothendieck ring of varieties, by considering varieties up to isomorphism instead of the more restrictive linear changes of coordinates. We give an explicit relation between the class of the affine hypersurface complement and the class of the projective hypersurface in the Grothendieck ring. As a consequence of the basic properties of algebro-geometric Feynman rules, we show in Proposition 2.9 that the stable birational equivalence class of the projective graph hypersurface of a non-1PI graph is equal to 1. We also discuss how the parametric formulation of Feynman integrals, in the case with nonzero mass and zero external momenta, may fit in the setting of algebro-geometric Feynman rules with values in the algebra of periods. The issue of the divergences of these integrals is further discussed in 4 . In $\$ 3$ we introduce a different algebro-geometric Feynman rule, that is obtained by mapping the ring $\mathcal{F}$ to the polynomial ring $\mathbb{Z}[T]$ via a morphism defined in terms of the Chern-Schwartz-MacPherson (CSM) characteristic classes of singular algebraic varieties. This morphism $\mathcal{F} \rightarrow \mathbb{Z}[T]$ does not factor through the usual Grothendieck ring of varieties $K_{0}\left(\mathcal{V}_{k}\right)$, as we show explicitly in Example 2.8. The main theorem showing the multiplicative property of this polynomial invariant over disjoint unions of graphs is stated in Theorem 3.6, and its proof is reduced in steps to a formula, given in Theorem 3.13, for the Chern-Schwartz-MacPherson classes of joins of disjoint subvarieties of projective space. In this same section, Proposition 3.1 lists the main properties of the polynomial invariant, including the fact that it recovers as a special value of the derivative the usual Euler characteristic of the projective hypersurface complements, thus effectively correcting for its failure to be a Feynman rule. A way to compute the coefficients of the polynomial invariant in terms of integrals of differential forms with logarithmic poles on a resolution is given in Remark 3.9. The following section, 84. discusses the BPHZ renormalization procedure, in the formulation of the ConnesKreimer theory in terms of Birkhoff factorization of characters of the Hopf algebra of Feynman graphs. Using the formulation in terms of characters with values in a RotaBaxter algebra of weight -1 , one can show that the BPHZ procedure can be applied to the various cases of algebro-geometric Feynman rules considered here. In $\$ 5$ we draw some analogies between the partition functions obtained by summing over graphs the algebro-geometric Feynman rules and the motivic zeta functions considered in the theory 
of motivic integration. Finally, 86 is devoted to the proof of Theorem 3.13. The main ingredients in the proof are Kwieciński's product formula and Yokura's Riemann-Roch theorem for CSM classes, together with a blow-up construction.

1.1. Feynman rules in quantum field theory. The Feynman rules prescribe that, in perturbative scalar quantum field theory, one assigns to a Feynman graph a formal (usually divergent) integral

$$
U(\Gamma, p)=C \int \frac{\delta\left(\sum_{i=1}^{n} \epsilon_{v, i} k_{i}+\sum_{j=1}^{N} \epsilon_{v, j} p_{j}\right)}{q_{1}\left(k_{1}\right) \cdots q_{n}\left(k_{n}\right)} \frac{d^{D} k_{1}}{(2 \pi)^{D}} \cdots \frac{d^{D} k_{n}}{(2 \pi)^{D}},
$$

where $C=\prod_{v \in V(\Gamma)} \lambda_{v}(2 \pi)^{D}$, with $\lambda_{v}$ the coupling constant of the monomial in the Lagrangian of degree equal to the valence of the vertex $v$. Here, $n=\# E_{\text {int }}(\Gamma)$, and $N=\# E_{\text {ext }}(\Gamma)$. The matrix $\epsilon_{v, i}$ is the incidence matrix of the (oriented) graph with entries $\epsilon_{v, i}= \pm 1$ if the edge $e_{i}$ is incident to the vertex $v$, outgoing or ingoing, and zero otherwise. The $q_{i}\left(k_{i}\right)$ are the Feynman propagators. The latter are quadratic forms, given in Euclidean signature by

$$
q_{i}\left(k_{i}\right)=k_{i}^{2}+m^{2},
$$

where $k_{i} \in \mathbb{R}^{D}$ is the momentum variable associated to the edge $e_{i}$ of the graph, with $k_{i}^{2}=\left\|k_{i}\right\|^{2}$ the Euclidean square norm in $\mathbb{R}^{D}$ and $m \geq 0$ the mass parameter. The integral $U(\Gamma, p)$ is a function of the external momenta $p=\left(p_{e}\right)_{e \in E_{e x t}(\Gamma)}$, where the $p_{e} \in \mathbb{R}^{D}$ satisfy the conservation law $\sum_{e \in E_{e x t}(\Gamma)} p_{e}=0$. The delta function in the numerator of (1.3) imposes linear relations at each vertex between the momentum variables, so that momentum conservation is preserved at each vertex. This reduces the number of independent variables of integration from the number of edges to the number of loops.

The form of the Feynman integral (1.3) immediately implies a multiplicative property. Namely, if the Feynman graph is a disjoint union $\Gamma=\Gamma_{1} \cup \Gamma_{2}$ of two components, then the integral satisfies

$$
U(\Gamma, p)=U\left(\Gamma_{1}, p_{1}\right) U\left(\Gamma_{2}, p_{2}\right),
$$

where $p_{i}$ are the external momenta of the graphs $\Gamma_{i}$, with $p=\left(p_{1}, p_{2}\right)$. This follows from the fact that there are no linear relations between the momentum variables assigned to edges in different connected components of the graph, so the integral splits as a product.

In quantum field theory one usually assembles the Feynman integrals of different graphs in a formal series that gives, for fixed external momenta $p=\left(p_{e}\right)=\left(p_{1}, \ldots, p_{N}\right)$, the Green function

$$
G(p)=\sum_{\Gamma} \frac{U(\Gamma, p)}{\# \operatorname{Aut}(\Gamma)}
$$

where $\operatorname{Aut}(\Gamma)$ are the symmetries of the graph. For a graph with several connected components, the symmetry factor behaves like

$$
\# \operatorname{Aut}(\Gamma)=\prod_{j}\left(n_{j}\right) ! \prod_{j} \# \operatorname{Aut}\left(\Gamma_{j}\right)^{n_{j}},
$$

where the $n_{j}$ are the multiplicities (i.e. there are $n_{j}$ connected components of $\Gamma$ all isomorphic to the same graph $\Gamma_{j}$ ). Thus, one can simplify the combinatorics of graphs in quantum field theory by considering only connected graphs and the corresponding connected Green functions.

One can further reduce the class of graphs that need to be considered, by passing to the 1PI effective action, where only the graphs that are "one-particle-irreducible" (1PI) are considered. These are the two-edge-connected graphs, namely those that cannot be disconnected by removal of a single edge. The reason why these suffice is again related 
to the multiplicative properties of Feynman rules. A connected graph $\Gamma$ can be described as a tree $T$ in which at the vertices one inserts 1 PI graphs $\Gamma_{v}$ with number of external edges equal to the valence of the vertex. The corresponding Feynman integral can then be written in the form of a product

$$
U(\Gamma, p)=\prod_{v \in T} U\left(\Gamma_{v}, p_{v}\right) \frac{1}{q_{e}\left(\left(p_{v}\right)_{e}\right)} \delta\left(\left(p_{v}\right)_{e}-\left(p_{v^{\prime}}\right)_{e}\right),
$$

i.e. a product of Feynman integrals for 1PI graphs and inverses of the propagators $q_{e}$ for the edges of the tree, with momenta matching the external momenta of the 1PI graphs.

When one takes the dimensional regularization of the Feynman integrals, one replaces the formal $U(\Gamma, p)$ by Laurent series, while maintaining the multiplicative properties over disjoint unions of graphs. Thus, if one defines a polynomial ring $\mathcal{H}$ generated by the 1PI graphs with the product corresponding to the disjoint union, the dimensionally regularized Feynman integral defines a ring homomorphism from $\mathcal{H}$ to the ring $\mathcal{R}$ of convergent Laurent series. When the polynomial ring $\mathcal{H}$ on the 1PI graphs is endowed with the Connes-Kreimer coproduct as in 10, and the ring of convergent Laurent series is endowed with the Rota-Baxter operator $\mathfrak{T}$ of projection onto the polar part, one can implement the BPHZ renormalization of the Feynman integral as in the Connes-Kreimer theory 10 as the Birkhoff factorization of the Feynman integrals $U(\Gamma, p)$ into a product of ring homomorphisms from $\mathcal{H}$ to $\mathfrak{T} \mathcal{R}$ and $(1-\mathfrak{T}) \mathcal{R}$, respectively defining the counterterms and the renormalized part of the Feynman integral.

In the following section we show how to abstract this setting to define algebro-geometric Feynman rules.

\section{Feynman Rules in algebraic geometry}

We give an abstract definition of Feynman rules which encompasses the case of Feynman integrals recalled above and that allows for algebro-geometric variants.

Definition 2.1. A Feynman rule is an assignment of an element $U(\Gamma)$ in a commutative ring $\mathcal{R}$ for each finite graph $\Gamma$, with the property that, for a disjoint union $\Gamma=\Gamma_{1} \cup \cdots \cup \Gamma_{k}$ of connected graphs $\Gamma_{i}$, the function behaves multiplicatively

$$
U(\Gamma)=U\left(\Gamma_{1}\right) \cdots U\left(\Gamma_{k}\right) .
$$

One also requires that, for a connected graph $\Gamma$ described as a finite tree $T$ with vertices replaced by 1 PI graphs $\Gamma_{v}$, the function $U(\Gamma)$ satisfies

$$
U(\Gamma)=U(L)^{\# E(T)} \prod_{v \in V(T)} U\left(\Gamma_{v}\right),
$$

where $L$ is the graph consisting of a single edge. Thus, a Feynman rule determines and is determined by a ring homomorphism $U: \mathcal{H} \rightarrow \mathcal{R}$, where $\mathcal{H}$ is the polynomial ring generated (over $\mathbb{Z}$ ) by the 1 PI graphs and by the assignment of the inverse propagator $U(L)$.

The definition we give here, which will suffice for our purposes, covers the original Feynman integrals only in the case where one neglects the external momenta (or sets them all to zero) and remains with a nontrivial propagator for external edges given only by the mass $m^{2}$. In fact, in that case, the formula (1.8) reduces to (2.2) with $U(L)=1 / \mathrm{m}^{2}$ for all the external edges of the graphs $\Gamma_{v}$. The property (2.1) is the multiplicative property of the Feynman rules (1.5). The dimensionally regularized Feynman integral $U(\Gamma)$ is described then in terms of a ring homomorphism $U: \mathcal{H} \rightarrow \mathcal{K}$ to the ring of convergent Laurent series, by identifying a monomial $\Gamma_{1} \cdots \Gamma_{k}$ in $\mathcal{H}$ with the disjoint union graph $\Gamma=\Gamma_{1} \cup \cdots \cup \Gamma_{k}$. 
We are especially interested in algebro-geometric Feynman rules associated to the parametric representation of Feynman integrals. In the parametric representation for a massless theory, one reformulates the integral (1.3) in the form

$$
U\left(\Gamma, p_{1}, \ldots, p_{N}\right)=C \frac{\Gamma\left(n-\frac{D \ell}{2}\right)}{(4 \pi)^{D \ell / 2}} \int_{\sigma_{n}} \frac{P_{\Gamma}(t, p)^{-n+D \ell / 2} \omega_{n}}{\Psi_{\Gamma}(t)^{-n+(\ell+1) D / 2}},
$$

where $t=\left(t_{1}, \ldots, t_{n}\right) \in \mathbb{A}^{n}$ with $n=\# E_{\text {int }}(\Gamma)$, integrated over the simplex $\sigma_{n}=\{t \in$ $\left.\mathbb{R}_{+}^{n} \mid \sum_{i} t_{i}=1\right\}$, with volume form $\omega_{n}$ and with $\Psi_{\Gamma}$ the Kirchhoff polynomial

$$
\Psi_{\Gamma}(t)=\operatorname{det} M_{\Gamma}(t), \quad \text { with } \quad\left(M_{\Gamma}(t)\right)_{r k}=\sum_{i} t_{i} \eta_{i r} \eta_{i k}
$$

where $\eta_{i k}$ is the circuit matrix of the graph (depending on a choice of orientation of the edges $e_{i}$ and of a basis $\left\{\ell_{k}\right\}$ of $\left.H_{1}(\Gamma)\right)$,

$$
\eta_{i k}=\left\{\begin{aligned}
+1 & \text { if edge } e_{i} \in \text { loop } \ell_{k}, \text { same orientation } \\
-1 & \text { if edge } e_{i} \in \text { loop } \ell_{k}, \text { reverse orientation } \\
0 & \text { if edge } e_{i} \notin \text { loop } \ell_{k} .
\end{aligned}\right.
$$

(This is equivalent to the definition given in the introduction.) If $b_{1}(\Gamma)=0$, we take $\Psi_{\Gamma}(t)=1$.

The function $P_{\Gamma}(t, p)$ is a homogeneus polynomial in $t$ of degree $b_{1}(\Gamma)+1$, which also has a definition in terms of the combinatorics of the graph. Notice that one can define parametric representations in the case of massive theories $m \neq 0$ as well and obtain a formulation similar to (2.3),

$$
U\left(\Gamma, p_{1}, \ldots, p_{N}\right)=C \frac{\Gamma\left(n-\frac{D \ell}{2}\right)}{(4 \pi)^{D \ell / 2}} \int_{\sigma_{n}} \frac{V_{\Gamma}(t, p)^{-n+D \ell / 2} \omega_{n}}{\Psi_{\Gamma}(t)^{D / 2}},
$$

where, however, $V_{\Gamma}(t, p)$ is no longer a homogeneous polynomial in $t$. Our definition of Feynman rules in Definition 2.1 is modeled on the massive case, because of the propagators $U(L)$ in (2.2). In both the massive and the massless case, at least for sufficiently large spacetime dimension $D$, in the range where $n \leq D \ell / 2$, the integral lives naturally on the complement in $\mathbb{A}^{n}$ of the affine hypersurface

$$
\hat{X}_{\Gamma}=\left\{t \in \mathbb{A}^{n} \mid \Psi_{\Gamma}(t)=0\right\} .
$$

In the massless case where both $\Psi_{\Gamma}$ and $P_{\Gamma}(t, p)$ in (2.3) are homogeneous polynomials, one usually reformulates the Feynman integral in terms of projective varieties and considers the complement $\mathbb{P}^{n-1} \backslash X_{\Gamma}$ of the projective hypersurface

$$
X_{\Gamma}=\left\{t=\left(t_{1}: \cdots: t_{n}\right) \in \mathbb{P}^{n-1} \mid \Psi_{\Gamma}(t)=0\right\},
$$

of which $\hat{X}_{\Gamma}$ is the affine cone. Although working in the projective setting is very natural (see 6], 8]), the discussion above indicates that, if one wants to accommodate both massless and massive theories, it is more natural to work in the affine setting. Moreover, we will see here that working with the affine hypersurfaces is better also from the point of view of having motivic Feynman rules.

Definition 2.2. An algebro-geometric Feynman rule is an invariant $\mathbb{U}(\Gamma)=\mathbb{U}\left(\mathbb{A}^{n} \backslash \hat{X}_{\Gamma}\right)$ of the graph hypersurface complement, with values in a commutative ring $\mathcal{R}$, with the following properties.

- For a disjoint union of graphs $\Gamma=\Gamma_{1} \cup \Gamma_{2}$, it satisfies $\mathbb{U}(\Gamma)=\mathbb{U}\left(\Gamma_{1}\right) \mathbb{U}\left(\Gamma_{2}\right)$.

- For a connected graph $\Gamma$ obtained from a finite tree $T$ and $1 P I$ graphs $\Gamma_{v}$ at the vertices $v \in V(T)$, it satisfies $\mathbb{U}(\Gamma)=\mathbb{U}(L)^{\# E(T)} \prod_{v \in V(T)} \mathbb{U}\left(\Gamma_{v}\right)$, where $\mathbb{U}(L)$ is the value on the line $L$, i.e. on the graph consisting of a single edge. 
An algebro-geometric Feynman rule is motivic if the invariant $\mathbb{U}(\Gamma)$ only depends on the class $\left[\mathbb{A}^{n} \backslash \hat{X}_{\Gamma}\right]$ of the hypersurface complement in the Grothendieck ring of varieties $K_{0}\left(\mathcal{V}_{\mathbb{Q}}\right)$.

By this definition, in particular, an algebro-geometric Feynman rule defines a ring homomorphism $\mathbb{U}: \mathcal{H} \rightarrow \mathcal{R}$ as in Definition 2.1 by interpreting a monomial $\Gamma_{1} \cdots \Gamma_{k}$ as the disjoint union $\Gamma=\Gamma_{1} \cup \cdots \cup \Gamma_{k}$. In the motivic case this homomorphism factors through the commutative ring $K_{0}\left(\mathcal{V}_{\mathbb{Q}}\right)$.

The dependence $\mathbb{U}(\Gamma)=\mathbb{U}\left(\mathbb{A}^{n} \backslash \hat{X}_{\Gamma}\right)$ of an algebro-geometric Feynman rule on the affine hypersurface complement should be understood here as a dependence on the variety $\mathbb{A}^{n} \backslash \hat{X}_{\Gamma}$ considered modulo linear changes of coordinates in $\mathbb{A}^{n}$. This will be explained more in detail in $\$ 2.1$ below. It will be then be clear from Lemma 2.3 that, unlike the case of general Feynman rules, the second property $\mathbb{U}(\Gamma)=\mathbb{U}(L)^{\# E(T)} \prod_{v \in V(T)} \mathbb{U}\left(\Gamma_{v}\right)$ in Definition 2.2 will in fact be a consequence of the multiplicativity $\mathbb{U}(\Gamma)=\mathbb{U}\left(\Gamma_{1}\right) \mathbb{U}\left(\Gamma_{2}\right)$ over disjoint unions $\Gamma=\Gamma_{1} \cup \Gamma_{2}$, together with the fact that the hypersurface complement does not distinguish between the case of the disjoint union $\Gamma=\Gamma_{1} \cup \Gamma_{2}$ and the case where the graphs $\Gamma_{1}$ and $\Gamma_{2}$ are joined at a single vertex.

Notice, moreover, that there are examples of combinatorially inequivalent connected 1PI graphs that have the same graph hypersurface, so that one can construct Feynman rules that are not algebro-geometric or motivic, by assigning different invariants to such graphs, so that the resulting ring homomorphism $\mathcal{H} \rightarrow \mathcal{R}$ does not factor through $K_{0}\left(\mathcal{V}_{k}\right)$ or through the ring $\mathcal{F}$ described in $\$ 2.1$ below.

2.1. A universal algebro-geometric Feynman rule. We show that algebro-geometric Feynman rules, in the sense of Definition 2.2 correspond to ring homomorphisms from a universal ring $\mathcal{F}$ to a given commutative ring. In particular, this defines a universal algebro-geometric Feynman rule obtained by assigning $\mathbb{U}(\Gamma)$ as the class of the hypersurface complement $\mathbb{A}^{n} \backslash \hat{X}_{\Gamma}$ in the ring $\mathcal{F}$. A motivic Feynman rule is then obtained by mapping $\mathcal{F}$ to the Grothendieck ring $K_{0}\left(\mathcal{V}_{\mathbb{Q}}\right)$.

We begin by the following simple observation, which explains why it is more convenient to work in the affine rather than the projective setting.

Lemma 2.3. For every graph $\Gamma$, let $X_{\Gamma} \subset \mathbb{P}^{n-1}$ be the projective hypersurface (2.8) and $\hat{X}_{\Gamma} \subset \mathbb{A}^{n}$ be its affine cone (2.7), with $n=\# E_{\text {int }}(\Gamma)$, as above.

Let $\Gamma=\Gamma_{1} \cup \Gamma_{2}$ be the union of two disjoint graphs. Then

$$
\mathbb{A}^{n_{1}+n_{2}} \backslash \hat{X}_{\Gamma}=\left(\mathbb{A}^{n_{1}} \backslash \hat{X}_{\Gamma_{1}}\right) \times\left(\mathbb{A}^{n_{2}} \backslash \hat{X}_{\Gamma_{2}}\right),
$$

where $n_{i}=\# E_{\text {int }}\left(\Gamma_{i}\right)$.

If neither $\Gamma_{1}$ nor $\Gamma_{2}$ is a forest, then the projective hypersurface complement $\mathbb{P}^{n_{1}+n_{2}-1} \backslash$ $X_{\Gamma}$ is a $\mathbb{G}_{m}$-bundle over the product $\left(\mathbb{P}^{n_{1}-1} \backslash X_{\Gamma_{1}}\right) \times\left(\mathbb{P}^{n_{2}-1} \backslash X_{\Gamma_{2}}\right)$ of the hypersurface complements of $\Gamma_{1}$ and $\Gamma_{2}$.

The same formulas hold if $\Gamma$ is obtained by attaching two disjoint graphs $\Gamma_{1}, \Gamma_{2}$ at a vertex.

Proof. It is clear from both the combinatorial definition recalled in the introduction, and from the definition (2.4) in terms of Kirchoff matrices $M_{\Gamma}(t)$, that if $\Gamma=\Gamma_{1} \cup \Gamma_{2}$ is a disjoint union (or if $\Gamma$ is obtained by attaching $\Gamma_{1}$ and $\Gamma_{2}$ at a vertex), then

$$
\Psi_{\Gamma}\left(t_{1}, \ldots, t_{n}\right)=\Psi_{\Gamma_{1}}\left(t_{1}, \ldots, t_{n_{1}}\right) \Psi_{\Gamma_{2}}\left(t_{n_{1}+1}, \ldots, t_{n_{1}+n_{2}}\right) .
$$

This says that $\hat{X}_{\Gamma_{1} \cup \Gamma_{2}}$ is the hypersurface in $\mathbb{A}^{n_{1}+n_{2}}$ obtained as the union

$$
\left(\hat{X}_{\Gamma_{1}} \times \mathbb{A}^{n_{2}}\right) \cup\left(\mathbb{A}^{n_{1}} \times \hat{X}_{\Gamma_{2}}\right),
$$

and formula (2.9) for the hypersurface complement $\mathbb{A}^{n_{1}+n_{2}} \backslash \hat{X}_{\Gamma}$ follows immediately. 
In projective terms, $X_{\Gamma}$ is given by the union of the cones $C^{n_{2}}\left(X_{\Gamma_{1}}\right), C^{n_{1}}\left(X_{\Gamma_{2}}\right)$ in $\mathbb{P}^{n_{1}+n_{2}-1}$ over $X_{\Gamma_{1}}$ and $X_{\Gamma_{2}}$, with vertices $\mathbb{P}^{n_{2}-1}$ and $\mathbb{P}^{n_{1}-1}$, respectively. Here one views the $\mathbb{P}^{n_{i}-1}$ containing $X_{\Gamma_{i}}$ as skew subspaces in $\mathbb{P}^{n_{1}+n_{2}-1}$. A point in the complement of $X_{\Gamma_{1} \cup \Gamma_{2}}$ in $\mathbb{P}^{n_{1}+n_{2}-1}$ is of the form

$$
\left(t_{1}: \cdots: t_{n_{1}+n_{2}}\right), \quad \text { where } \Psi_{\Gamma_{1}}\left(t_{1}, \cdots, t_{n_{1}}\right) \neq 0 \text { and } \Psi_{\Gamma_{2}}\left(t_{n_{1}+1}, \cdots, t_{n_{1}+n_{2}}\right) \neq 0 .
$$

Note that if $\Psi_{\Gamma_{1}} \not \equiv 1$ and $\Psi_{\Gamma_{2}} \not \equiv 1$, then $\Psi_{\Gamma_{1}}\left(t_{1}: \cdots: t_{n_{1}}\right) \neq 0$ only if $\left(t_{1}: \cdots: t_{n_{1}}\right) \neq 0$, and $\Psi_{\Gamma_{2}}\left(t_{n_{1}+1}: \cdots: t_{n_{1}+n_{2}}\right) \neq 0$ only if $\left(t_{n_{1}+1}: \cdots: t_{n_{1}+n_{2}}\right) \neq 0$. This says that if neither $\Gamma_{1}$ nor $\Gamma_{2}$ is a forest, then we have a regular map

$$
\left(\mathbb{P}^{n_{1}+n_{2}-1} \backslash X_{\Gamma_{1} \cup \Gamma_{2}}\right) \rightarrow\left(\mathbb{P}^{n_{1}-1} \backslash X_{\Gamma_{1}}\right) \times\left(\mathbb{P}^{n_{2}-1} \backslash X_{\Gamma_{2}}\right)
$$

given by

$$
\left(t_{1}: \cdots: t_{n_{1}}: t_{n_{1}+1}: \cdots: t_{n_{1}+n_{2}}\right) \mapsto\left(\left(t_{1}: \cdots: t_{n_{1}}\right),\left(t_{n_{1}+1}: \cdots: t_{n_{1}+n_{2}}\right)\right) \quad .
$$

This map is evidently surjective, and the fiber over $\left(\left(t_{1}: \cdots: t_{n_{1}}\right),\left(t_{n_{1}+1}: \cdots: t_{n_{1}+n_{2}}\right)\right)$ consists of the points

$$
\left(u t_{1}: \cdots: u t_{n_{1}}: v t_{n_{1}+1}: \cdots: v t_{n_{1}+n_{2}}\right)
$$

with $(u: v) \in \mathbb{P}^{1}, u v \neq 0$. These fibers are tori $\mathbb{G}_{m}(k)=k^{*}$, completing the proof.

If either $\Gamma_{1}$ or $\Gamma_{2}$ is a forest, the corresponding hypersurface $X_{\Gamma_{i}}$ is empty; the map (2.10) is not defined everywhere in this case.

The observation above implies that, if we want to construct Feynman rule $\mathbb{U}(\Gamma)$ in terms of the hypersurface complements, then by working in the affine setting it suffices to have an invariant of affine varieties that is multiplicative on products and behaves in the natural way with respect to complements, that is, it satisfies an inclusion-exclusion property. This indicates that the natural target of algebro-geometric Feynman rules should be a ring reminiscent of the Grothendieck ring of varieties $K_{0}\left(\mathcal{V}_{k}\right)$. However, it will be advantageous to work in a ring with a more rigid equivalence relation than in the definition of $K_{0}\left(\mathcal{V}_{k}\right)$ : this will be a ring mapping to $K_{0}\left(\mathcal{V}_{k}\right)$, but also carrying enough information to allow us to define Feynman rules by means of characteristic classes of immersed varieties. The natural receptacle of our algebro-geometric Feynman rules will be the Grothendieck ring of immersed conical varieties, which we define as follows.

Definition 2.4. Let $\mathcal{F}$ be the ring whose elements are formal finite integer linear combinations of equivalence classes of closed conical (that is, defined by homogeneous ideals) reduced algebraic sets $V$ of $\mathbb{A}^{\infty}$, such that $V \subseteq \mathbb{A}^{N}$ for some finite $N$, modulo the equivalence relation given by linear changes of coordinates, and with the further relation dictating 'inclusion-exclusion':

$$
[V \cup W]=[V]+[W]-[V \cap W] .
$$

The ring structure is given by the product induced by

$$
[V] \cdot[W]=[V \times W] .
$$

This is an embedded version of the Grothendieck ring, and it maps to the Grothendieck ring since varieties differing by a linear change of coordinates are isomorphic. It will also map to polynomial rings, via characteristic classes of complements, as we will explain in 3 .

If $U$ is a locally closed set, defined as the complement $V \backslash W$ of two closed conical subsets, we can define a class

$$
[U]:=[V]-[W]
$$

in $\mathcal{F}$; the inclusion-exclusion property guarantees that this assignment is independent of the specific representation of $U$, and that the product formula (2.12) extends to locally 
closed sets. This implies that ring homomorphisms $\mathcal{F} \rightarrow \mathcal{R}$ of the Grothendieck ring of immersed conical varieties to arbitrary commutative rings define algebro-geometric Feynman rules:

Proposition 2.5. Let $I: \mathcal{F} \rightarrow \mathcal{R}$ be a ring homomorphism to a commutative ring $\mathcal{R}$. For every graph $\Gamma$ with $n=\# E_{\text {int }}(\Gamma)$, define $\mathbb{U}(\Gamma) \in R$ by

$$
\mathbb{U}(\Gamma):=I\left(\left[\mathbb{A}^{n}\right]\right)-I\left(\left[\hat{X}_{\Gamma}\right]\right)=I\left(\left[\mathbb{A}^{n} \backslash \hat{X}_{\Gamma}\right]\right) .
$$

Then $\mathbb{U}$ is multiplicative under disjoint unions: if $\Gamma_{1}, \Gamma_{2}$ are disjoint graphs, then $\mathbb{U}\left(\Gamma_{1} \cup\right.$ $\left.\Gamma_{2}\right)=\mathbb{U}\left(\Gamma_{1}\right) \cdot \mathbb{U}\left(\Gamma_{2}\right)$.

The same formula holds if $\Gamma_{1}, \Gamma_{2}$ share a single vertex. Moreover, if $\Gamma$ is obtained by connecting two disjoint graphs $\Gamma_{1}, \Gamma_{2}$ by an edge, then the invariant satisfies

$$
\mathbb{U}(\Gamma)=\mathbb{U}\left(\Gamma_{1}\right) \mathbb{U}(L) \mathbb{U}\left(\Gamma_{2}\right),
$$

where $\mathbb{U}(L)$ is the invariant associated to the graph $L$ consisting of a single edge. This is given by $\mathbb{U}(L)=I\left(\left[\mathbb{A}^{1}\right]\right)=: \mathfrak{L}$, the value of $I$ on the class of the affine line.

Proof. The claims are all preserved under homomorphisms, so it suffices to prove them for the invariant $\mathbb{U}$ with values in $\mathcal{F}$ defined by

$$
\mathbb{U}(\Gamma):=\left[\mathbb{A}^{n} \backslash \hat{X}_{\Gamma}\right] \in \mathcal{F}
$$

for a graph $\Gamma$ with $n$ internal edges. The multiplicativity under disjoint unions, and under the operation of attaching graphs at a single vertex, follows then immediately from formula (2.9) in Lemma 2.3. In turn, formula (2.14) follows from the multiplicativity. To see that $\mathbb{U}(L)=\left[\mathbb{A}^{1}\right]$, simply recall that the graph hypersurface corresponding to a single edge (or to any forest) is $\emptyset$.

We denote here by $\mathfrak{L}$ the value $I\left(\left[\mathbb{A}^{1}\right]\right)$, as this will map to the Lefschetz motive $\mathbb{L}$ in the Grothendieck group. Note that we then have $I\left(\left[\mathbb{A}^{n}\right]\right)=\mathfrak{L}^{n}$; by definition, this is the invariant associated with any forest with $n$ edges, since the graph polynomial of a forest is 1 (and hence the corresponding graph hypersurface is empty). In particular, $1=I\left(\left[\mathbb{A}^{0}\right]\right)=\mathbb{U}(\star)$ is the invariant of the trivial graph $\star$ consisting of one vertex and no edges.

We have given in Definition 2.1 an equivalent characterization of Feynman rules in terms of a ring homomorphism $\mathbb{U}: \mathcal{H} \rightarrow \mathcal{R}$ together with an "inverse propagator" $\mathbb{U}(L)$. An algebro-geometric Feynman rule defined as above by a ring homomorphism $I: \mathcal{F} \rightarrow \mathcal{R}$ corresponds, in these terms, to the homomorphism $\mathbb{U}: \mathcal{H} \rightarrow \mathcal{R}$ obtained by precomposition with the ring homomorphism

$$
\mathcal{H} \rightarrow \mathcal{F}, \quad \Gamma \mapsto\left[\mathbb{A}^{n}\right]-\left[\hat{X}_{\Gamma}\right],
$$

for all connected 1PI graphs $\Gamma$, and with the inverse propagator $\left[\mathbb{A}^{1}\right] \in \mathcal{F}$.

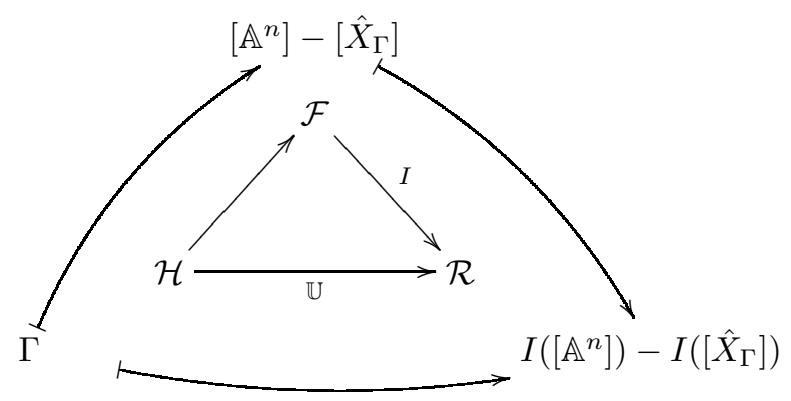


By Proposition 2.5 we have a 'universal' algebro-geometric Feynman rule given by the identity homomorphism $I: \mathcal{F} \rightarrow \mathcal{F}$. Again, this corresponds to the ring homomorphism $\mathcal{H} \rightarrow \mathcal{F}$ that assigns $\left[\mathbb{A}^{n}\right]-\left[\hat{X}_{\Gamma}\right]$ to a connected 1 PI graph with $n=\# E_{\text {int }}(\Gamma)$ and with inverse propagator $\left[\mathbb{A}^{1}\right] \in \mathcal{F}$.

2.2. Operations on graphs and Feynman rules. The universal algebro-geometric Feynman rule defined by $\left[\mathbb{A}^{n}\right]-\left[\hat{X}_{\Gamma}\right]$ in $\mathcal{F}$ satisfies the following properties for elementary geometric operations on a graph. These properties are inherited by any other algebrogeometric Feynman rule.

- Let $\Gamma^{\prime}$ be obtained from $\Gamma$ by attaching an edge to a vertex of $\Gamma$. Then

$$
\mathbb{U}\left(\Gamma^{\prime}\right)=\left[\mathbb{A}^{1}\right] \cdot \mathbb{U}(\Gamma)
$$

- Let $\Gamma$ be a graph that is not $1 \mathrm{PI}$. Then $\mathbb{U}(\Gamma)$ is of the form

$$
\mathbb{U}(\Gamma)=\left[\mathbb{A}^{1}\right] \cdot B
$$

for some class $B \in \mathcal{F}$.

- Let $\Gamma^{\prime}$ be obtained from $\Gamma$ by splitting an edge. Then

$$
\mathbb{U}\left(\Gamma^{\prime}\right)=\left[\mathbb{A}^{1}\right] \cdot \mathbb{U}(\Gamma)
$$

- Let $\Gamma^{\prime}$ be obtained from $\Gamma$ by attaching a looping edge to a vertex. Then

$$
\mathbb{U}\left(\Gamma^{\prime}\right)=\left(\left[\mathbb{A}^{1}\right]-1\right) \cdot \mathbb{U}(\Gamma)
$$

- Let $\Gamma$ be an $n$-side polygon. Then

$$
\mathbb{U}(\Gamma)=\left[\mathbb{A}^{n}\right]-\left[\mathbb{A}^{n-1}\right] .
$$

All these properties follow very easily from the definition of $\mathbb{U}(\Gamma)$. For instance, the property for non-1PI graphs follows directly from (2.14), while attaching a looping edge amounts to multiplying the equation of the graph hypersurface by a new variable, and viewing the result in a space of dimension 1 higher. In affine space, and in terms of the complement, this is clearly the same as taking a product by $\mathbb{A}^{1} \backslash \mathbb{A}^{0}$.

2.3. Motivic Feynman rules. The $\operatorname{ring} \mathcal{F}$ maps to the Grothendieck ring of varieties $K_{0}\left(\mathcal{V}_{k}\right)$ by mapping the equivalence class $[X] \in \mathcal{F}$ under linear coordinate changes to the isomorphism class $[X] \in K_{0}\left(\mathcal{V}_{k}\right)$. It is a ring homomorphism since the product is in both cases defined by the class of the product of manifolds. Thus, one obtains in this way a motivic Feynman rule defined by $\Gamma \mapsto\left[\mathbb{A}^{n} \backslash \hat{X}_{\Gamma}\right] \in K_{0}\left(\mathcal{V}_{k}\right)$. This corresponds to the ring homomorphism $\mathbb{U}: \mathcal{H} \rightarrow K_{0}\left(\mathcal{V}_{k}\right)$ that maps the monomial $\Gamma_{1} \cdots \Gamma_{k}$, where the $\Gamma_{i}$ are $1 \mathrm{PI}$ graphs, to the class

$$
\mathbb{U}\left(\Gamma_{1} \cdots \Gamma_{k}\right)=\left[\mathbb{A}^{n} \backslash \hat{X}_{\Gamma}\right]=\left[\mathbb{A}^{n_{1}} \backslash \hat{X}_{\Gamma_{1}}\right] \cdots\left[\mathbb{A}^{n_{k}} \backslash \hat{X}_{\Gamma_{k}}\right],
$$

where $\Gamma=\Gamma_{1} \cup \cdots \cup \Gamma_{k}$ is the disjoint union and $n=\sum_{i} n_{i}$. The inverse propagator is $\mathbb{U}(L)=\mathbb{L}=\left[\mathbb{A}^{1}\right]$, the Lefschetz motive, i.e. the class of the affine line in $K_{0}\left(\mathcal{V}_{k}\right)$.

This means that one can think of the "propagator" as being the formal inverse $\mathbb{L}^{-1}$ of the Lefschetz motive. This corresponds to the Tate motive $\mathbb{Q}(1)$ when one maps in the natural way (see [17]) the Grothendieck ring of varieties $K_{0}\left(\mathcal{V}_{k}\right)$ to the Grothendieck ring of motives $K_{0}\left(\mathcal{M}_{k}\right)$.

The relation between the motivic Feynman rule (2.15) and the hypersurface complement in projective space is described as follows.

Lemma 2.6. If $\Gamma$ is not a forest, the hypersurface complements $\mathbb{A}^{n} \backslash \hat{X}_{\Gamma}$ and $\mathbb{P}^{n-1} \backslash X_{\Gamma}$ are related in the Grothendieck ring $K_{0}\left(\mathcal{V}_{k}\right)$ by

$$
\left[\mathbb{A}^{n} \backslash \hat{X}_{\Gamma}\right]=(\mathbb{L}-1)\left[\mathbb{P}^{n-1} \backslash X_{\Gamma}\right] .
$$


Proof. We have

$$
\left[\hat{X}_{\Gamma}\right]=(\mathbb{L}-1)\left[X_{\Gamma}\right]+1,
$$

since $\hat{X}_{\Gamma}$ is the affine cone in $\mathbb{A}^{n}$ over $X_{\Gamma}$. Thus, we obtain

$$
\begin{aligned}
{\left[\mathbb{A}^{n} \backslash \hat{X}_{\Gamma}\right] } & =\mathbb{L}^{n}-1-(\mathbb{L}-1)\left[X_{\Gamma}\right] \\
& =(\mathbb{L}-1)\left(\mathbb{L}^{n-1}+\cdots+\mathbb{L}+1-\left[X_{\Gamma}\right]\right) \\
& =(\mathbb{L}-1)\left(\left[\mathbb{P}^{n-1}\right]-\left[X_{\Gamma}\right]\right) .
\end{aligned}
$$

Thus, we see that the factor $(\mathbb{L}-1)$ restores the multiplicative property of Feynman rules that is not satisfied at the level of the projective hypersurface complements.

Example 2.7. The graph hypersurfaces corresponding to the so-called banana graphs are studied in [3]. Lemma 2.6 and formula (3.13) in [3] yield that

$$
\left[\mathbb{A}^{n} \backslash \hat{X}_{\Gamma_{n}}\right]=(\mathbb{L}-1) \frac{(\mathbb{L}-1)^{n}-(-1)^{n}}{\mathbb{L}}+n(\mathbb{L}-1)^{n-1},
$$

where $\Gamma_{n}$ denotes the $n$-th banana graph ( $n$ parallel edges joining two vertices).

Given the motivic Feynman rule determined by the ring homomorphism $\mathbb{U}: \mathcal{H} \rightarrow$ $K_{0}\left(\mathcal{V}_{k}\right)$, with $\mathbb{U}(\Gamma)=\left[\mathbb{A}^{n} \backslash \hat{X}_{\Gamma}\right]$ and inverse propagator $\mathbb{L}$, one can obtain other motivic Feynman rules with values in commutative rings $\mathcal{R}$ using motivic measures. Recall that a motivic measure is by definition a ring homomorphism $\mu: K_{0}\left(\mathcal{V}_{k}\right) \rightarrow \mathcal{R}$ (see for instance [23], §1.3), so that the composite $\mu \circ \mathbb{U}$ defines an $\mathcal{R}$-valued motivic Feynman rule.

Notice that, in particular, when one considers the ring homomorphism from $K_{0}\left(\mathcal{V}_{k}\right)$ to $\mathbb{Z}$ given by the ordinary topological Euler characteristic, the image of the classes $\left[\mathbb{A}^{n} \backslash \hat{X}_{\Gamma}\right]$ is zero if $\Gamma$ is not a forest, as one can see from the presence of the torus factor $\left[\mathbb{G}_{m}\right]=\mathbb{L}-1$ in (2.16), while if one computes the Euler characteristic of the projective hypersurface complements $\left[\mathbb{P}^{n-1} \backslash X_{\Gamma}\right]$ this will in general be non-zero (see for instance the examples computed in [3]) but the multiplicative property of Feynman rules is no longer satisfied. We show in 3 below how one can define an algebro-geometric Feynman rule that assigns a polynomial invariant in $\mathbb{Z}[T]$ to the class in $\mathcal{F}$ of each hypersurface complement $\mathbb{A}^{n} \backslash \hat{X}_{\Gamma}$, in such a way that the value at zero of the derivative of the polynomial recovers the Euler characteristic of the complement of the projective hypersurface $X_{\Gamma}$. This invariant will be our best answer to the question of a generalization of the ordinary Euler characteristic that satisfies the multiplicative property of Feynman rules and from which the usual Euler characteristic can be recovered as a special value. This invariant is not obtained from a homomorphism $K_{0}\left(\mathcal{V}_{k}\right) \rightarrow \mathbb{Z}[T]$ as the following example shows.

Example 2.8. The two graphs
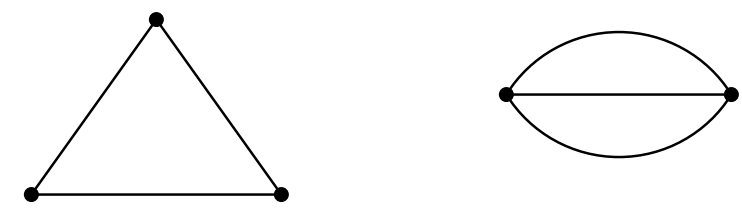

have the same motivic invariant $\left[\mathbb{A}^{3}\right]-\left[\mathbb{A}^{2}\right]$, but different polynomial invariants: $T(T+1)^{2}$ and $T\left(T^{2}+T+1\right)$, respectively.

It is proved in 23 that the quotient of the Grothendieck ring $K_{0}\left(\mathcal{V}_{\mathbb{C}}\right)$ by the ideal generated by $\mathbb{L}=\left[\mathbb{A}^{1}\right]$ is isomorphic as a ring to $\mathbb{Z}[S B]$, the ring of the multiplicative monoid $S B$ of stable birational equivalence classes of varieties in $\mathcal{V}_{\mathbb{C}}$. Recall that two 
(irreducible) varieties $X$ and $Y$ are stably birationally equivalent if $X \times \mathbb{P}^{n}$ and $Y \times \mathbb{P}^{m}$ are birationally equivalent for some $n, m \geq 0$. The observations of $\$ 2.2$ above then give the following.

Proposition 2.9. Let $\Gamma$ be a graph that is not 1PI. Then the stable birational equivalence class of the projective graph hypersurface satisfies $\left[X_{\Gamma}\right]_{s b}=1$ in $\mathbb{Z}[S B]$.

Proof. We know by Lemma 2.6 that, in the Grothendieck ring $K_{0}\left(\mathcal{V}_{\mathbb{C}}\right)$, we have $\left[\mathbb{A}^{n} \backslash \hat{X}_{\Gamma}\right]=$ $\mathbb{L}^{n}-1-(\mathbb{L}-1)\left[X_{\Gamma}\right]$. Moreover, by the observation made in $\$ 2.2$ we know that for a graph $\Gamma$ that is not $1 \mathrm{PI}$ the class $\left[\mathbb{A}^{n} \backslash \hat{X}_{\Gamma}\right]=\mathbb{L} \cdot\left[\mathbb{A}^{n-1} \backslash \hat{X}_{\Gamma^{\prime}}\right]$, where $\Gamma^{\prime}$ is the graph obtained from $\Gamma$ by removing a disconnecting edge $L$ and $\mathbb{L}=\left[\mathbb{A}^{1}\right]=\mathbb{U}(L)$. Then we use the fact that $\mathbb{Z}[S B]=K_{0}\left(\mathcal{V}_{\mathbb{C}}\right) /(\mathbb{L})$ as in $\left[23\right.$, and we obtain that $\left[\mathbb{A}^{n} \backslash \hat{X}_{\Gamma}\right]_{s b}=0 \in \mathbb{Z}[S B]$, while $\mathbb{L}^{n}-1-(\mathbb{L}-1)\left[X_{\Gamma}\right] \in K_{0}\left(\mathcal{V}_{\mathbb{C}}\right)$ becomes $-1+\left[X_{\Gamma}\right]_{s b} \in \mathbb{Z}[S B]$, so that we obtain $\left[X_{\Gamma}\right]_{s b}-1=0 \in \mathbb{Z}[S B]$.

A variant of the motivic Feynman rule (2.15) is obtained by setting

$$
\mathbb{U}(\Gamma)=\frac{\left[\mathbb{A}^{n} \backslash \hat{X}_{\Gamma}\right]}{\mathbb{L}^{n}},
$$

with values in the ring $K_{0}\left(\mathcal{V}_{k}\right)\left[\mathbb{L}^{-1}\right]$, where one inverts the Lefschetz motive. Dividing by $\mathbb{L}^{n}$ has the effect of normalizing the "Feynman integral" $\mathbb{U}(\Gamma)$ by the value it would have if $\Gamma$ were a forest on the same number of edges. For the original Feynman integrals this would measure the amount of linear dependence between the edge momentum variables created by the presence of the interaction vertices. We will discuss in $\$ 4$ some advantages of using the motivic Feynman rule (2.18) as opposed to (2.15).

Moreover, notice that, modulo the important problem of divergences of the Feynman integral, which needs to be treated via a suitable regularization and renormalization procedure, which in the algebro-geometric setting often involves blowups of the divergence locus (see [8]), one would like to think of the original Feynman rule given by the parametric Feynman integral as an algebro-geometric Feynman rule with values in the algebra $\mathcal{P}$ of periods. Recall that conjecturally (see 21]) the algebra $\mathcal{P}$ of periods is generated over $\mathbb{Q}$ by equivalence classes of the form $[(X, D, \omega, \sigma)]$, where $X$ is a smooth affine variety over $\mathbb{Q}, D \subset X$ is a normal crossings divisor, $\omega \in \Omega^{\operatorname{dim}(X)}(X)$ is an algebraic differential form, and $\sigma \in H_{\operatorname{dim}(X)}(X(\mathbb{C}), D(\mathbb{C}) ; \mathbb{Q})$ is a relative homology class. The equivalence relation is taken modulo the change of variables formula and the Stokes formula for integrals (see 21] for more details). In the setting that we are considering, where in Feynman integrals we set the external momenta equal to zero and keep a non-zero mass, so that the Feynman rules satisfy (2.1) and (2.2), the function $V_{\Gamma}(t, p)$ in the numerator of the parametric Feynman integral (2.6) is reduced to $\left.V(t, p)\right|_{p=0}=m^{2}$. This follows from the fact that, in general, $V_{\Gamma}(t, p)$ is of the form

$$
V_{\Gamma}(t, p)=p^{\dagger} R_{\Gamma}(t) p+m^{2}
$$

where $R_{\Gamma}(t)$ is another matrix associated to the graph $\Gamma$ defined in terms of cut-sets, whose explicit expression we do not need here (the interested reader can see for instance [18] or [5]). Thus, for the massive case with zero external momenta, the parametric Feynman integral is, up to a multiplicative constant and a possibly divergent $\Gamma$-factor, of the form

$$
\int_{\sigma_{n}} \frac{\omega_{n}}{\Psi_{\Gamma}(t)^{D / 2}}
$$

Modulo the important issue of divergences coming from the nontrivial intersections $\sigma_{n} \cap$ $\hat{X}_{\Gamma}$, we can then think of the original Feynman rule as a morphism to the algebra of 
periods $\mathcal{P}$ that assigns

$$
\mathbb{U}(\Gamma)=\left[\left(\mathbb{A}^{n} \backslash \hat{X}_{\Gamma}, \hat{\Sigma}_{n}, \Psi_{\Gamma}^{-D / 2} \omega_{n}, \sigma_{n}\right)\right],
$$

where $\hat{\Sigma}_{n}=\left\{t \in \mathbb{A}^{n} \mid \prod_{i} t_{i}=0\right\}$.

A possible way to handle the divergences in terms of "integrating around the singularities" using Leray coboundaries was proposed in [25]. We discuss briefly in $\$$ how this might fit with Feynman rules of the form (2.20).

\section{Characteristic Classes and Feynman Rules}

In this section we define a ring homomorphism

$$
I_{C S M}: \mathcal{F} \rightarrow \mathbb{Z}[T]
$$

and hence (by Proposition 2.5) obtain a polynomial valued Feynman rule. We will denote by $C_{\Gamma}(T)$ the invariant corresponding to $I_{C S M}$ for a graph $\Gamma$ : that is,

$$
C_{\Gamma}(T):=I_{C S M}\left(\mathbb{A}^{n}\right)-I_{C S M}\left(\hat{X}_{\Gamma}\right)
$$

if $\Gamma$ has $n$ (internal) edges.

This invariant will carry information related to the Chern-Schwartz-MacPherson (CSM) class of the graph hypersurface of a given graph $\Gamma$. The reader is addressed to $\S 2.2$ of [3] for a quick review of the definition and basic properties of these classes.

Before defining $I_{C S M}$, we highlight a few features of the invariant.

Proposition 3.1. Let $\Gamma$ be a graph with $n$ edges.

- $C_{\Gamma}(T)$ is a monic polynomial of degree $n$.

- If $\Gamma$ is a forest, then $C_{\Gamma}(T)=(T+1)^{n}$. In particular, the inverse propagator corresponds to $T+1$.

- If $\Gamma$ is not a forest, then $C_{\Gamma}(T)$ is a multiple of $T$.

- The coefficient of $T^{n-1}$ in $C_{\Gamma}(T)$ equals $n-b_{1}(\Gamma)$.

- The value $C_{\Gamma}^{\prime}(0)$ of the derivative of $C_{\Gamma}(T)$ at 0 equals the Euler characteristic of the complement $\mathbb{P}^{n} \backslash X_{\Gamma}$.

The proof of this lemma will follow the statement of Theorem 3.6 .

Of course, the invariant will also satisfy the properties listed in 22.2 , These take the following form:

- Let $\Gamma^{\prime}$ be obtained from $\Gamma$ by attaching an edge to a vertex of $\Gamma$, or by splitting an edge of $\Gamma$. Then

$$
C_{\Gamma^{\prime}}(T)=(T+1) \cdot C_{\Gamma}(T)
$$

- Let $\Gamma^{\prime}$ be obtained from $\Gamma$ by attaching a looping edge to a vertex. Then

$$
C_{\Gamma^{\prime}}(T)=T \cdot C_{\Gamma}(T)
$$

- Let $\Gamma$ be a graph that is not 1 PI. Then $C_{\Gamma}(-1)=0$.

- Let $\Gamma$ be an $n$-side polygon. Then

$$
C_{\Gamma}(T)=T(T+1)^{n-1} .
$$

Remark 3.2. The parallel between the motivic invariant introduced in 92.3 is even more apparent if one changes the variable $T$ to $L=T+1$. We choose $T$ because it has a compelling geometric interpretation: $T^{k}$ corresponds to the class $\left[\mathbb{P}^{k}\right]$ in the ambient space which we use to define the invariant. Ultimately, this is due to the fact that the CSM class of a torus $\mathbb{T}^{k}$ embedded in $\mathbb{P}^{k}$ as the complement of the 'algebraic symplex' is $\left[\mathbb{P}^{k}\right]$, cf. Theorem 4.2 in $[1]$. 
In order to define $I_{C S M}$, it suffices to define it on a set of generators of $\mathcal{F}$, and verify that the definition preserves the relations defining $\mathcal{F}$.

Generators for $\mathcal{F}$ consist of conical subvarieties $\hat{X} \subseteq \mathbb{A}^{N}$. View $\hat{X}$ as a locally closed subset of $\mathbb{P}^{N}$; as such, it determines a CSM class in the Chow group $A\left(\mathbb{P}^{N}\right)$ of $\mathbb{P}^{N}$ :

$$
c_{*}\left(\mathbb{1}_{\hat{X}}\right)=a_{0}\left[\mathbb{P}^{0}\right]+\cdots+a_{N}\left[\mathbb{P}^{N}\right] .
$$

(Here $\mathbb{1}$ denotes the constant function 1 on the specified locus; we denote by $c_{*}$ MacPherson's natural transformation relating constructible functions and classes in the Chow group.)

Definition 3.3. We define

$$
G_{\hat{X}}(T):=a_{0}+a_{1} T+\cdots+a_{N} T^{N}
$$

Example 3.4. For $\hat{X}=\mathbb{A}^{N}$ :

$$
G_{\mathbb{A}^{N}}(T):=(T+1)^{N}
$$

Indeed, viewing $\mathbb{A}^{N}$ as the complement of $\mathbb{P}^{N-1}$ in $\mathbb{P}^{N}$ gives

$c_{*}\left(\mathbb{1}_{\mathbb{A}^{N}}\right)=c_{*}\left(\mathbb{1}_{\mathbb{P}^{N}}\right)-c_{*}\left(\mathbb{1}_{\mathbb{P}^{N-1}}\right)=\left((1+H)^{N+1}-H(1+H)^{N}\right) \cap\left[\mathbb{P}^{N}\right]=(1+H)^{N} \cap\left[\mathbb{P}^{N}\right]$, where $H$ denotes the hyperplane class in $\mathbb{P}^{N}$. The coefficient of $\left[\mathbb{P}^{k}\right]$ in this class is $\left(\begin{array}{l}N \\ k\end{array}\right)$, with the stated result.

Remark 3.5. Here are a few comments on the definition of $G_{\hat{X}}(T)$.

- The definition does not depend on the dimension of the ambient affine space $\mathbb{A}^{N}$ : the largest $i$ for which $a_{i} \neq 0$ is the dimension of $\hat{X}$.

- If $\hat{X}$ and $\hat{X}^{\prime}$ differ by a coordinate change, then clearly $G_{\hat{X}}(T)=G_{\hat{X}^{\prime}}(T)$.

- If $\hat{X}, \hat{Y} \subseteq \mathbb{A}^{N}$, then

$$
G_{\hat{X} \cup \hat{Y}}(T)=G_{\hat{X}}(T)+G_{\hat{Y}}(T)-G_{\hat{X} \cap \hat{Y}}(T) \quad:
$$

this follows from the inclusion-exclusion property of CSM classes.

- By the previous two points, the definition goes through the equivalence relation defining $\mathcal{F}$. Thus, we can define a map $I_{C S M}: \mathcal{F} \rightarrow \mathbb{Z}[T]$ by setting

$$
I_{C S M}([\hat{X}]):=G_{\hat{X}}(T),
$$

and extending by linearity. This map is clearly a group homomorphism.

We claim that:

Theorem 3.6. $I_{C S M}$ is a homomorphism of rings.

Once Theorem 3.6 is established, Proposition 2.5 will show that setting

$$
C_{\Gamma}(T)=U_{C S M}(\Gamma):=I_{C S M}\left(\left[\mathbb{A}^{n}\right]\right)-I_{C S M}\left(\left[\hat{X}_{\Gamma}\right]\right)=G_{\mathbb{A}^{n}}(T)-G_{\hat{X}_{\Gamma}}(T),
$$

where $n=$ the number of edges of $\Gamma$, defines a multiplicative graph invariant. The polynomial $C_{\Gamma}(t)$ satisfies all the properties listed at the beginning of this subsection:

Proof of Proposition 3.1. Since $\hat{X}_{\Gamma}$ is properly contained in $\mathbb{A}^{n}$, the dominant term in the difference $G_{\mathbb{A}^{n}}(T)-G_{\hat{X}_{\Gamma}}(T)$ is $T^{n}$ : this proves the first point.

If $\Gamma$ is a forest, then $\hat{X}_{\Gamma}=\emptyset$. Thus $C_{\Gamma}(T)=G_{\mathbb{A}^{n}}(T)=(T+1)^{n}$ (Example 3.4), proving the second point.

If $\Gamma$ is not a forest, $\hat{X}_{\Gamma} \neq \emptyset$, and the Euler characteristic of $\hat{X}_{\Gamma}$ is $1\left(\hat{X}_{\Gamma}\right.$ is an affine cone). Therefore the constant term of $G_{\mathbb{A}^{n}}(T)-G_{\hat{X}_{\Gamma}}(T)$ is $1-1=0$ : this proves that $C_{\Gamma}(T)$ is a multiple of $T$ in this case, as claimed.

As to the fourth point: if $\Gamma$ is a forest, then $b_{1}(\Gamma)=0$ and the formula is verified. Thus, assume $\Gamma$ is not a forest. The coefficient of $T^{n-1}$ in $G_{\mathbb{A}^{n}}(T)=(T+1)^{n}$ is $n$, while the 
coefficient of $T^{n-1}$ in $G_{\hat{X}_{\Gamma}}(T)$ equals the coefficient of the top-dimensional term $\left[\mathbb{P}^{n-1}\right]$ in $c_{*}\left(\hat{X}_{\Gamma}\right)$. This equals the degree of the hypersurface $X_{\Gamma}$, which is $b_{1}(\Gamma)$, and the formula follows.

Finally, $C_{\Gamma}^{\prime}(0)$ equals the coefficient of $T$ in $C_{\Gamma}(T)$. If $\Gamma$ is a forest, then $C_{\Gamma}(T)=$ $(T+1)^{n}$, so this coefficient is $n$, and equals the Euler characteristic of $\mathbb{P}^{n-1} \backslash \emptyset$. If $\Gamma$ is not a forest, then the coefficient of $T$ in $C_{\Gamma}(T)$ equals the coefficient of $\left[\mathbb{P}^{0}\right]$ in the ChernSchwartz-MacPherson class of $\mathbb{P}^{n-1} \backslash X_{\Gamma}$ (see Proposition 3.7 below). This equals the Euler characteristic of $\mathbb{P}^{n-1} \backslash X_{\Gamma}$, by general properties of Chern-Schwartz-MacPherson classes (see for example [3], §2.2).

As in the motivic case, the invariant $C_{\Gamma}(T)$ can be expressed in terms of the complement of the projective graph hypersurface. The analog of Lemma 2.6 is:

Proposition 3.7. If $\Gamma$ is not a forest, and has $n$ edges, then

$$
c_{*}\left(\mathbb{1}_{\mathbb{P}^{n-1} \backslash X_{\Gamma}}\right)=H^{n} C_{\Gamma}(1 / H) \cap\left[\mathbb{P}^{n-1}\right],
$$

where $H$ is the hyperplane class in $\mathbb{P}^{n-1}$. Otherwise put, if $\Gamma$ is not a forest, then $C_{\Gamma}(T)$ may be recovered from $c_{*}\left(\mathbb{1}_{\mathbb{P}^{n-1} \backslash X_{\Gamma}}\right)$ by replacing the class $\left[\mathbb{P}^{k}\right]$ in $c_{*}\left(\mathbb{1}_{\mathbb{P}^{n-1} \backslash X_{\Gamma}}\right)$ by $T^{k+1}$.

Proof. Indeed, if $c_{*}(X)=f(H) \cap\left[\mathbb{P}^{n-1}\right]$, then

$$
c_{*}\left(\mathbb{1}_{\mathbb{A}^{n} \backslash \hat{X}_{\Gamma}}\right)=c_{*}\left(\mathbb{A}^{n}\right)-c_{*}\left(\hat{X}_{\Gamma}\right)=\left((1+H)^{n}-f(H)-H^{n}\right) \cap\left[\mathbb{P}^{n}\right] \quad:
$$

this follows from a straightforward computation, using the formula for the CSM class of a cone (Proposition 5.2 in [3]). Formula (3.1) says that $H^{n} C_{\Gamma}(1 / H)=(1+H)^{n}-f(H)-H^{n}$. On the other hand, the polynomial $(1+H)^{n}-H^{n}-f(H)$ corresponds to $c_{*}\left(\mathbb{1}_{\mathbb{P}^{n-1} \backslash X_{\Gamma}}\right)$ in $\mathbb{P}^{n-1}$; this is precisely the statement.

Example 3.8. For $\Gamma_{n}=$ the $n$-edge banana graph,

$$
C_{\Gamma}(T)=T(T-1)^{n-1}+n T^{n-1} .
$$

Indeed, Remark 4.11 in [3] gives

$$
c_{*}\left(\mathbb{1}_{\mathbb{P}^{n-1} \backslash X_{\Gamma}}\right)=\left((1-H)^{n-1}+n H\right) \cap\left[\mathbb{P}^{n-1}\right] .
$$

By Proposition 3.7, therefore,

$$
H^{n} C_{\Gamma}(1 / H)=(1-H)^{n-1}+n H
$$

and the result follows at once.

Remark 3.9. Let $\Gamma$ be a graph with $n$ edges, that is not a forest, and suppose

$$
C_{\Gamma}(T)=T^{n}+a_{n-2} T^{n-1}+\cdots+a_{0} T .
$$

Let $\pi: \widetilde{\mathbb{P}}^{n-1} \rightarrow \mathbb{P}^{n-1}$ be a proper birational map such that $D:=\pi^{-1}\left(X_{\Gamma}\right)$ is a divisor with normal crossings and nonsingular components. Then

$$
a_{k}=\int\left(\pi^{*} H\right)^{k} \cdot c\left(T_{\widetilde{\mathbb{P}}^{n-1}}(-\log D)\right) \cap\left[\widetilde{\mathbb{P}}^{n-1}\right],
$$

where $T_{\widetilde{\mathbb{P}} n-1}(-\log D)$ denotes the dual of the bundle $\Omega_{\widetilde{\mathbb{P}} n-1}^{1}(\log D)$ of differential forms with logarithmic poles along $D$, and $\int \alpha$ stands for the degree of the class $\alpha$, in the sense of [15, Definition 1.4.

This follows immediately from Proposition 3.7 and the expression of $c_{*}\left(\mathbb{1}_{\mathbb{P}^{n-1} \backslash \hat{X}_{\Gamma}}\right)$ in terms of Chern classes of logarithmic forms (cf. [3], §2.3). 
In the rest of this section we reduce the proof of Theorem 3.6 to a statement (Theorem 3.13) concerning Chern-Schwartz-MacPherson classes of joins of disjoint subvarieties of projective space. The proof of this statement will be deferred to 6 .

In order to prove Theorem 3.6 , it suffices to prove that

$$
G_{\hat{X} \times \hat{Y}}(T)=G_{\hat{X}}(T) \cdot G_{\hat{Y}}(T)
$$

for all conical affine varieties $\hat{X} \subseteq \mathbb{A}^{m}, \hat{Y} \subseteq \mathbb{A}^{n}$. If $\hat{X}=\emptyset$ or $\hat{Y}=\emptyset$, this is immediate, as this identity is $0=0$ in this case. If $\hat{X}$ or $\hat{Y}$ is a point (that is, the origin of the ambient affine space), the identity is also immediate. Indeed, $G_{\mathbb{A}^{0}}(T)=1$.

Therefore:

Lemma 3.10. In order to prove Theorem 3.6, it suffices to prove that if $\hat{X} \subseteq \mathbb{A}^{m}$, resp. $\hat{Y} \subseteq \mathbb{A}^{n}$ are affine cones over projective algebraic sets $X \subseteq \mathbb{P}^{m-1}$, resp. $Y \subseteq \mathbb{P}^{n-1}$, then

$$
G_{\hat{X} \times \hat{Y}}(T)=G_{\hat{X}}(T) \cdot G_{\hat{Y}}(T)
$$

What is a little surprising now is that this is not obvious. There is a "product formula for CSM classes', due to Kwieciński ([22, [1]); but it relates classes in $\mathbb{P}^{m}, \mathbb{P}^{n}$ to classes in $\mathbb{P}^{m} \times \mathbb{P}^{n}$, while the polynomial $G(T)$ refers to a class in $\mathbb{P}^{m+n}$. While $\mathbb{P}^{m} \times \mathbb{P}^{n}$ and $\mathbb{P}^{m+n}$ can be related in a straightforward way by blow-ups and blow-downs, tracking the fate of CSM classes across blow-up operations is in itself a nontrivial (and worthy) task. One might optimistically think that if a locally closed set avoids the center of a blow-up, then the CSM class of its preimage ought to be the pull-back of its CSM class; this is not true in general, as simple examples show. The fact that it is true in certain cases is what we prove in 3, Corollary 4.4, and this result is crucial for the computation of CSM classes of graph hypersurfaces of banana graphs. We know of no general result of the same type handling the present situation, so we have to provide a rather ad-hoc argument to prove the formula in Lemma 3.10. Kwieciński's product formula will be an ingredient in our proof.

By Lemma 3.10, we are reduced to dealing with affine cones over (nonempty) projective varieties $X \subseteq \mathbb{P}^{m-1}$. We begin by relating $G_{\hat{X}}(T)$ to the CSM class of $X$.

Lemma 3.11. Let $X \subseteq \mathbb{P}^{m-1}$ be a nonempty subvariety, and let $f(h)$ be the polynomial of degree $<m$ in the hyperplane class $h$ of $\mathbb{P}^{m-1}$, such that

$$
c_{*}\left(\mathbb{1}_{X}\right)=f(h) \cap\left[\mathbb{P}^{m-1}\right] .
$$

Then

$$
h^{m} G_{\hat{X}}(1 / h)=f(h)+h^{m} .
$$

Proof. Consider the cone $C(X)$ of $X \subseteq \mathbb{P}^{m-1}$ in $\mathbb{P}^{m}$. By Proposition 5.2 in [3],

$$
c_{*}\left(\mathbb{1}_{C(X)}\right)=\left((1+h) f(h)+h^{m}\right) \cap\left[\mathbb{P}^{m}\right],
$$

where $h$ denotes the hyperplane class in $\mathbb{P}^{m}$. Since $\hat{X} \subseteq \mathbb{A}^{m}$ may be realized as the complement of $X$ in $C(X)$,

$$
c_{*}\left(\mathbb{1}_{\hat{X}}\right)=\left((1+h) f(h)+h^{m}-h f(h)\right) \cap\left[\mathbb{P}^{m}\right]=\left(f(h)+h^{m}\right) \cap\left[\mathbb{P}^{m}\right] .
$$

Since $h^{k} \cap\left[\mathbb{P}^{m-k}\right]$ corresponds to $T^{m-k}$ in Definition 3.3 ,

$$
f(h)+h^{m}=h^{m} G_{\hat{X}}(1 / h)
$$

as stated.

Next, we relate the affine product of varieties to the projective join. View $\mathbb{P}^{m-1}, \mathbb{P}^{n-1}$ as disjoint subspaces of $\mathbb{P}^{m+n-1}$. For $X \subseteq \mathbb{P}^{m-1}, Y \subseteq \mathbb{P}^{n-1}$, we will denote by $J(X, Y)$ (the 'join' of $X$ and $Y$ ) the union of all lines connecting points of $X$ to points of $Y$ in $\mathbb{P}^{m+n-1}$. 
Lemma 3.12. The product $\hat{X} \times \hat{Y} \subseteq \mathbb{A}^{m+n}$ is the affine cone over the join $J(X, Y) \subseteq$ $\mathbb{P}^{m+n-1}$.

Proof. Denote by $\left(x_{1}: \ldots: x_{m}: y_{1}: \ldots: y_{n}\right)$ the points of $\mathbb{P}^{m+n-1}$; identify $\mathbb{P}^{m-1}$ with the set of points $\left(x_{1}: \ldots: x_{m}: 0: \ldots: 0\right)$ and $\mathbb{P}^{n-1}$ with the set of points $\left(0: \ldots: 0: y_{1}: \ldots: y_{n}\right)$. If $\left(x_{1}: \ldots: x_{m}\right) \in X$ and $\left(y_{1}: \ldots: y_{n}\right) \in Y$, the points of the line in $\mathbb{P}^{m+n-1}$ joining these two points are all and only the points

$$
\left(s x_{1}: \ldots: s x_{m}: t y_{1}: \ldots: t y_{n}\right)
$$

as $(s: t)$ varies in $\mathbb{P}^{1}$. It follows that a point $\left(x_{1}: \ldots: x_{m}: y_{1}: \ldots: y_{n}\right)$ is a point of $J(X, Y)$ if and $\left(x_{1}: \ldots: x_{m}\right)$ satisfies the homogeneous ideal of $X$ in $\mathbb{P}^{m-1}$ and $\left(y_{1}: \ldots: y_{n}\right)$ satisfies the homogeneous ideal of $Y$ in $\mathbb{P}^{n-1}$. This is the case if and only if

$$
\left(x_{1}, \ldots, x_{m}, y_{1}, \ldots, y_{n}\right) \in \hat{X} \times \hat{Y} \subseteq \mathbb{A}^{m+n},
$$

and this shows that the affine cone over $J(X, Y)$ is $\hat{X} \times \hat{Y}$.

By Lemma 3.12, the sought-for formula in Lemma 3.10 may be rewritten as

$$
G_{\widehat{J(X, Y)}}(T)=G_{\hat{X}}(T) \cdot G_{\hat{Y}}(T) \quad ;
$$

or, equivalently (after a change of variable and harmless manipulations):

$$
H^{m+n} G_{\widehat{J(X, Y)}}(1 / H)-H^{m+n}=H^{m} G_{\hat{X}}(1 / H) \cdot H^{n} G_{\hat{Y}}(1 / H)-H^{m+n}
$$

for all nonempty $X \subseteq \mathbb{P}^{m-1}, Y \subseteq \mathbb{P}^{n-1}$. Here $H$ is simply a variable; but the two sides of the identity are polynomials of degree $<(m+n)$ in $H$, so formula (3.2) may be verified by interpreting $H$ as the hyperplane class in $\mathbb{P}^{m+n-1}$. This formulation and Lemma 3.11 reduce the proof of Theorem 3.6 to the following computation of the CSM class of a join:

Theorem 3.13. Let $\mathbb{P}^{m-1}, \mathbb{P}^{n-1}$ be disjoint subspaces of $\mathbb{P}^{m+n-1}$, and let $X \subseteq \mathbb{P}^{m-1}$, $Y \subseteq \mathbb{P}^{n-1}$ be nonempty subvarieties. Let $f(H)$, resp. $g(H)$ be polynomials such that

$$
c_{*}\left(\mathbb{1}_{X}\right)=H^{n} f(H) \cap\left[\mathbb{P}^{m+n-1}\right] \quad, \quad c_{*}\left(\mathbb{1}_{Y}\right)=H^{m} g(H) \cap\left[\mathbb{P}^{m+n-1}\right] .
$$

Then

$$
c_{*}\left(\mathbb{1}_{J(X, Y)}\right)=\left(\left(f(H)+H^{m}\right)\left(g(H)+H^{n}\right)-H^{m+n}\right) \cap\left[\mathbb{P}^{m+n-1}\right] .
$$

This is a result of independent interest, and its proof is deferred to 46 As argued in this section, Theorem 3.13 establishes Theorem 3.6, concluding the proof that $C_{\Gamma}(T)$ satisfies the Feynman rules and the other properties listed in this section.

\section{Renormalization for algebro-geometric Feynman rules}

The Connes-Kreimer theory [10] (see also a detailed account in $\S 1$ of [12]) shows that the BPHZ procedure of renormalization of dimensionally regularized Feynman integrals can be formulated as a Birkhoff factorization in the affine group scheme dual to the ConnesKreimer Hopf algebra of Feynman graphs. The explicit recursive formula for the Birkhoff factorization proved by Connes and Kreimer in [10] gives a multiplicative splitting of an algebra homomorphism $U: \mathcal{H} \rightarrow \mathcal{K}$, with $\mathcal{K}$ the field of convergent Laurent series, as

$$
U=\left(U_{-} \circ S\right) \star U_{+}
$$

where $S$ is the antipode in the Connes-Kreimer Hopf algebra $\mathcal{H}$ and $U_{ \pm}: \mathcal{H} \rightarrow \mathcal{K}_{ \pm}$are algebra homomorphisms with values, respectively, in the algebra of convergent power series $\mathcal{K}_{+}$and the polynomial algebra $\mathcal{K}_{-}=\mathbb{C}\left[z^{-1}\right]$. The product $\star$ is dual to the coproduct $\Delta$ of $\mathcal{H}$ by $\left(U_{1} \star U_{2}\right)(X)=\left(U_{1} \otimes U_{2}\right)(\Delta(X))$.

The proof that the $U_{ \pm}$, given in [10] by a recursive formula, are algebra homomorphisms uses the Rota-Baxter identity satisfied by the operator of projection of a Laurent series onto its polar part. The argument of Connes-Kreimer can therefore be easily generalized, 
in essentially the same form (see [14), to the case of algebra homomorphisms $U: \mathcal{H} \rightarrow \mathcal{R}$, with $\mathcal{H}$ a polynomial ring in the 1 PI graphs and $\mathcal{R}$ a Rota-Baxter ring of weight -1 . We recall briefly how the renormalization procedure works.

A Rota-Baxter ring of weight $\lambda$ is a commutative ring $\mathcal{R}$ endowed with a linear operator $\mathfrak{T}: \mathcal{R} \rightarrow \mathcal{R}$ satisfying the Rota-Baxter identity

$$
\mathfrak{T}(x) \mathfrak{T}(y)=\mathfrak{T}(x \mathfrak{T}(y))+\mathfrak{T}(\mathfrak{T}(x) y)+\lambda \mathfrak{T}(x y) .
$$

Such an operator is called a Rota-Baxter operator of weight $\lambda$.

Let $\mathcal{H}$ denote the polynomial ring generated over $\mathbb{Z}$ by the 1 PI graphs, endowed with the coproduct

$$
\Delta(\Gamma)=\Gamma \otimes 1+1 \otimes \Gamma+\sum_{\gamma \subset \Gamma} \gamma \otimes \Gamma / \gamma
$$

Here the proper subgraphs $\gamma \subset \Gamma$ are possibly multiconnected, with components that are 1PI. This is just slightly different from the Connes-Kreimer coproduct in as we are not fixing a Lagrangian for a scalar field theory, hence we do not restrict only to subgraphs such that $\Gamma / \gamma$ is still a Feynman graph of the given theory. In this sense, it is similar to the Hopf algebras of graphs considered in [19] 27. The ring $\mathcal{H}=\oplus_{n \geq 0} \mathcal{H}_{n}$ is graded by the number $n=\# E_{\text {int }}(\Gamma)$ of internal edges of the graph and the antipode is defined inductively as

$$
S(\Gamma)=-\Gamma-\sum_{\gamma \subset \Gamma} S(\gamma) \Gamma / \gamma .
$$

We then have the following result of Connes-Kreimer [10] (see also [14 for the formulation in Rota-Baxter terms).

Proposition 4.1. Suppose given a ring homomorphism $U: \mathcal{H} \rightarrow \mathcal{R}$, with $\mathcal{H}$ as above and $\mathcal{R}$ a Rota-Baxter ring of weight -1 . Let $\mathcal{R}_{-}$denote the ring obtained by adjoining a unit to the ring $\mathfrak{T} \mathcal{R}$ and let $\mathcal{R}_{+}$be the ring $\mathcal{R}_{+}=(1-\mathfrak{T}) \mathcal{R}$. Then the recursive formulae

$$
\begin{gathered}
U_{-}(\Gamma)=-\mathfrak{T}\left(U(\Gamma)+\sum_{\gamma \subset \Gamma} U_{-}(\gamma) U(\Gamma / \gamma)\right) \\
U_{+}(\Gamma)=(1-\mathfrak{T})\left(U(\Gamma)+\sum_{\gamma \subset \Gamma} U_{-}(\gamma) U(\Gamma / \gamma)\right)
\end{gathered}
$$

determine a Birkhoff factorization (4.1) into algebra homomorphisms $U_{ \pm}: \mathcal{H} \rightarrow \mathcal{R}_{ \pm}$. There is a unique such factorization satisfying the normalization condition $\epsilon_{-} \circ U_{-}=\epsilon$, where $\epsilon_{-}: \mathcal{R}_{-} \rightarrow \mathbb{Z}$ is the augmentation and $\epsilon$ is the counit of $\mathcal{H}$, defined by $\epsilon(X)=0$ for $\operatorname{deg}(X)>0$.

In the case of the dimensionally regularized Feynman integrals, the $U_{-}$gives the counterterms and the evaluation of the convergent power series $U_{+}(\Gamma)$,

$$
\left.U_{+}(\Gamma)\right|_{z=0},
$$

gives the renormalized value of the Feynman integral $U(\Gamma)$.

We can apply the same procedure to the algebro-geometric Feynman rules, using suitable Rota-Baxter operators on the target ring. This will give new multiplicative invariants of graphs obtained by following the same BPHZ procedure that governs the renormalization of divergent Feynman integrals.

For example, consider the motivic Feynman rule $\mathbb{U}(\Gamma)=\left[\mathbb{A}^{n} \backslash \hat{X}_{\Gamma}\right] \mathbb{L}^{-n}$ in $K_{0}\left(\mathcal{V}_{\mathbb{C}}\right)\left[\mathbb{L}^{-1}\right]$. In the ring $K_{0}\left(\mathcal{V}_{\mathbb{C}}\right)\left[\mathbb{L}^{-1}\right]$ we can still consider the Rota-Baxter operator of projection onto the polar part (in the variable $\mathbb{L})$. The renormalized Feynman rule $\mathbb{U}_{+}(\Gamma)$ defined as in 
(4.5) defines a class in $K_{0}\left(\mathcal{V}_{\mathbb{C}}\right)$ and the "renormalized value of the Feynman integral" (4.6) defines a class in $\mathbb{Z}[S B]$,

$$
\left.\mathbb{U}_{+}(\Gamma)\right|_{\mathbb{L}=0}=\left.(1-\mathfrak{T})\left(U(\Gamma)+\sum_{\gamma \subset \Gamma} U_{-}(\gamma) U(\Gamma / \gamma)\right)\right|_{\mathbb{L}=0} \in \mathbb{Z}[S B]=K_{0}\left(\mathcal{V}_{\mathbb{C}}\right) /(\mathbb{L}) .
$$

Notice that the parts of $\left[\mathbb{A}^{n} \backslash \hat{X}_{\Gamma}\right],\left[\mathbb{A}^{n_{1}} \backslash \hat{X}_{\gamma}\right]$ and $\left[\mathbb{A}^{n_{2}} \backslash \hat{X}_{\Gamma / \gamma}\right]$ that are contained in the ideal $(\mathbb{L}) \subset K_{0}\left(\mathcal{V}_{0}\right)$ contribute cancellations to the $\mathbb{L}^{n}$ in the denominator. It is possible that this invariant and the Birkhoff factorization of $\mathbb{U}(\Gamma)$ may help to detect the presence of non-mixed-Tate strata in the graph hypersurface $X_{\Gamma}$ coming from the contributions of hypersurfaces of smaller graphs $\gamma \subset \Gamma$ or quotient graphs $\Gamma / \gamma$,

For invariants like $C_{\Gamma}(T)$ that take values in polynomial rings, one can consider different kinds of Rota-Baxter operators. For example, consider the basis of $\mathbb{Q}[T]$ as a $\mathbb{Q}$-vector space, given by the polynomials

$$
\pi_{n}(T)=\frac{T(T+1) \cdots(T+n-1)}{n !}, \quad \forall n \geq 1, \quad \pi_{0}(T)=1 .
$$

The linear operator $\mathfrak{T}\left(\pi_{n}\right)=\pi_{n+1}$ is a non-trivial Rota-Baxter operator of weight -1 on the polynomial ring $\mathbb{Q}[T]$ (see [16]). One can then apply the BPHZ procedure with respect to this or other interesting Rota-Baxter operators to have a Birkhoff factorization of the given invariant with respect to an assigned Rota-Baxter structure. We do not pursue further in this paper the meaning of BPHZ with respect to different possible Rota-Baxter operators, but we only remark that algebro-geometric Feynman rules provide a supply of examples where one can abstractly study the properties of the BPHZ renormalization procedure. For example, the question of whether the Grothendieck ring of varieties $K_{0}\left(\mathcal{V}_{k}\right)$ or our Grothendieck ring of immersed conical varieties $\mathcal{F}$ admit a Rota-Baxter structure of weight -1 appears to be a problem of independent interest.

Finally, we can consider again the possible definition (2.20) of Feynman rules with values in the algebra $\mathcal{P}$ of periods and the problem of the divergences caused by the nontrivial intersections of the domain of integration $\sigma_{n}$ with the hypersurface $\hat{X}_{\Gamma}$. In 25 a regularization for Feynman integrals of the form (2.19) was proposed based on replacing the part of the integral that takes place in a neighborhood of the hypersurface $\hat{X}_{\Gamma}$ of the form $D_{\epsilon}\left(\hat{X}_{\Gamma}\right)=\cup_{s \in \Delta_{\epsilon}^{*}} \hat{X}_{\Gamma}(s)$, given by the level sets $\hat{X}_{\Gamma}(s)=\left\{t \in \mathbb{A}^{n} \mid \Psi_{\Gamma}(t)=s\right\}$ for $s \in \Delta_{\epsilon}^{*}$ a small punctured disk of radius $\epsilon>0$, with an integral on a Leray coboundary $\mathcal{L}_{\epsilon}\left(\sigma_{n}\right)=\pi^{-1}\left(\sigma_{n} \cap X_{\epsilon}\right)$, for $\pi_{\epsilon}: \partial D_{\epsilon}\left(\hat{X}_{\Gamma}\right) \rightarrow \hat{X}_{\Gamma}(\epsilon)$ the circle bundle projection. This has the effect of replacing the (divergent) integration on the locus $\sigma_{n} \cap \hat{X}_{\Gamma}$ with one on circles around the singular locus. By the results of [4 Part III, $\S 10.2$ and Theorem 4.4 of [25], the resulting integral $\mathbb{U}(\Gamma)(\epsilon)$ extends to a meromorphic function of $\epsilon$ in a small neighborhood of $\epsilon=0$, with a pole at $\epsilon=0$. One can then apply the BPHZ renormalization procedure, with $\mathfrak{T}$ the projection onto the polar part of the Laurent series in $\epsilon$ and obtain a renormalized $\mathbb{U}_{+}(\Gamma)$.

\section{The Partition Function and TAte motives}

In quantum field theory it is customary to consider the full partition function of the theory, arranged in an asymptotic series by loop number or another suitable grading of the Hopf algebra of Feynman graphs, instead of looking only at the contribution of individual Feynman graphs. Besides the loop number $\ell=b_{1}(\Gamma)$, other suitable gradings $\delta(\Gamma)$ are given by the number $n=\# E_{\text {int }}(\Gamma)$ of internal edges, or by $\# E_{\text {int }}(\Gamma)-b_{1}(\Gamma)=\# V(\Gamma)-b_{0}(\Gamma)$, the number of vertices minus the number of connected components (cf. [12] p.77). 
When one considers motivic Feynman rules, these partition functions appear to be interesting analogs of the motivic zeta functions considered in [20], 23]. For instance, one can consider a partition function given by the formal series

$$
Z(t)=\sum_{N \geq 0} \sum_{\delta(\Gamma)=N} \frac{\mathbb{U}(\Gamma)}{\# \operatorname{Aut}(\Gamma)} t^{N}
$$

where $\delta(\Gamma)$ is any one of the gradings on the Hopf algebra of Feynman graphs described above and where $\mathbb{U}(\Gamma)=\left[\mathbb{A}^{n} \backslash \hat{X}_{\Gamma}\right] \in K_{0}\left(\mathcal{V}_{k}\right)$. Given a motivic measure $\mu: K_{0}\left(\mathcal{V}_{k}\right) \rightarrow \mathcal{R}$, this gives a zeta function with values in $\mathcal{R}[[t]]$ of the form

$$
Z_{\mathcal{R}}(t)=\sum_{N \geq 0} \sum_{\delta(\Gamma)=N} \frac{\mu(\mathbb{U}(\Gamma))}{\# \operatorname{Aut}(\Gamma)} t^{N}
$$

Of particular interest, in view of the recent results of [7], is the case where one restricts the class of graphs to connected graphs without looping edges and without multiple edges and takes the grading $\delta(\Gamma)=\# V(\Gamma)-b_{0}(\Gamma)$. In this case, one is considering the zeta function

$$
Z(t)=\sum_{N \geq 1} \frac{t^{N}}{N !} \sum_{\# V(\Gamma)=N} \mathbb{U}(\Gamma) \frac{N !}{\# \operatorname{Aut}(\Gamma)} .
$$

The result of [7] shows that

$$
S_{N}=\sum_{\# V(\Gamma)=N}\left[X_{\Gamma}\right] \frac{N !}{\# \operatorname{Aut}(\Gamma)}
$$

is in the Tate part of the Grothendieck ring, $S_{N} \in \mathbb{Z}[\mathbb{L}]$. It then follows that the zeta function $Z(t)$ above takes values in $\mathbb{Z}[\mathbb{L}][[t]]$.

One can investigate the behaviour of these "motivic zeta functions" by the same techniques used in 23 to study the original motivic zeta function defined by Kapranov in [20].

\section{THE FORMUlA FOR CSM CLASSES OF JOINS}

This section is devoted to the proof of Theorem 3.13. We first recall the statement.

Let $X \subseteq \mathbb{P}^{m-1}, Y \subseteq \mathbb{P}^{n-1}$ be nonempty subvarieties, and view $\mathbb{P}^{m-1}, \mathbb{P}^{n-1}$ as disjoint subspaces of $\mathbb{P}^{m+n-1}$. The task is to compute the push-forward to $\mathbb{P}^{m+n-1}$ of the ChernSchwartz-MacPherson class of the join $J(X, Y)$, defined as the union of the lines in $\mathbb{P}^{m+n-1}$ connecting all points of $X$ to all points of $Y$. The class will be expressed as a polynomial in the class $H$ of a hyperplane in $\mathbb{P}^{m+m-1}$, obtained in terms of the polynomials similarly giving the Chern-Schwartz-MacPherson classes of $X$ in $\mathbb{P}^{m-1}, Y$ in $\mathbb{P}^{n-1}$.

We will denote by $h$ the hyperplane class in $\mathbb{P}^{m-1}$, and by $k$ the hyperplane class in $\mathbb{P}^{n-1}$. Let $f(h), g(k)$ be polynomials of degree $<m$, resp. $<n$, such that

$$
\begin{gathered}
c_{*}\left(\mathbb{1}_{X}\right)=f(h) \cap\left[\mathbb{P}^{m-1}\right], \\
c_{*}\left(\mathbb{1}_{Y}\right)=g(k) \cap\left[\mathbb{P}^{n-1}\right] .
\end{gathered}
$$

Theorem 3.13 states the following result:

$$
c_{*}\left(\mathbb{1}_{J(X, Y)}\right)=\left(\left(f(H)+H^{m}\right)\left(g(H)+H^{n}\right)-H^{m+n}\right) \cap\left[\mathbb{P}^{m+n-1}\right] .
$$

The rest of this section is devoted to the proof of this formula.

Example 6.1. If $Y=\mathbb{P}^{n-1}$, then $J(X, Y)$ is the cone $C^{n}(X)$ on $X$, with vertex $\mathbb{P}^{n-1}$. Since $c\left(T \mathbb{P}^{n-1}\right)=(1+H)^{n}-H^{n}$, (6.1) gives

$$
c_{*}\left(C^{n}(X)\right)=\left((1+H)^{n}\left(f(H)+H^{m}\right)-H^{m+n}\right) \cap\left[\mathbb{P}^{m+n-1}\right] .
$$


where a push-forward is understood. In particular, for $n-1=0$ (so $C^{1}(X)=C(X)$ is just an 'ordinary' cone in projective space) this agrees with the formula for cones given in Proposition 5.2 of $[3$.

Example 6.2. For $X=\mathbb{P}^{m-1}, Y=\mathbb{P}^{n-1}$, the join $J(X, Y)$ is the whole of $\mathbb{P}^{m+n-1}$. Theorem 3.13 gives

$$
c_{*}\left(\mathbb{P}^{m+n-1}\right)=\left((1+H)^{m}(1+H)^{n}-H^{m+n}\right) \cap\left[\mathbb{P}^{m+n-1}\right]
$$

as it should.

We will realize the join of $X$ and $Y$ as a projection of a $\mathbb{P}^{1}$-bundle over $X \times Y$. Consider the rational map

$$
\mathbb{P}^{m+n-1} \rightarrow \mathbb{P}^{m-1} \times \mathbb{P}^{n-1}
$$

given by

$$
\left(x_{1}: \ldots: x_{m}: y_{1}: \ldots: y_{n}\right) \mapsto\left(\left(x_{1}: \ldots: x_{m}\right),\left(y_{1}: \ldots: y_{n}\right)\right) \quad ;
$$

this is well-defined away from the union $\mathbb{P}^{m-1} \cup \mathbb{P}^{n-1}$ consisting of points where either

$$
y_{1}=\cdots=y_{n}=0
$$

or

$$
x_{1}=\cdots=x_{m}=0 .
$$

Letting $B \ell$ be the blow-up of $\mathbb{P}^{m+n-1}$ along these two linear subspaces, we obtain a diagram

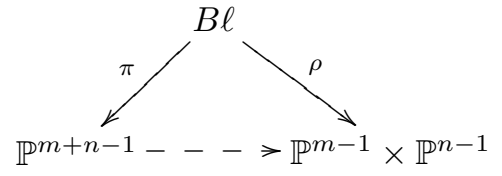

resolving the given rational map, and realizing $B \ell$ as a $\mathbb{P}^{1}$-bundle over $\mathbb{P}^{m-1} \times \mathbb{P}^{n-1}$. Concretely, $\rho^{-1}(p, q)$ may be identified with the (proper transform of the) line in $\mathbb{P}^{m+n-1}$ joining $p \in \mathbb{P}^{m-1}$ to $q \in \mathbb{P}^{n-1}$.

Summary of the argument: we will use Kwieciński's product formula (22]) and Yokura's Riemann-Roch for Chern-Schwartz-MacPherson classes (29) to compute the class of the inverse image of $X \times Y$ in $B \ell$. The formula for the class of $J(X, Y)$ will follow from this and the basic functoriality property of CSM classes.

We first collect the necessary ingredients.

As noted above, $h$ and $k$ denote respectively the hyperplane classes in $\mathbb{P}^{m-1}, \mathbb{P}^{n-1}$; we use the same letters to denote their pull-backs to the product $\mathbb{P}^{m-1} \times \mathbb{P}^{n-1}$, and to $B \ell$.

Lemma 6.3. With notation as above,

$$
c_{*}\left(\mathbb{1}_{X \times Y}\right)=f(h) g(k) \cap\left[\mathbb{P}^{m-1} \times \mathbb{P}^{n-1}\right] .
$$

Proof. There is a natural map $A_{*} X \otimes A_{*} Y \rightarrow A_{*}(X \times Y)(\S 1.10$ in [15). By Kwieciński's theorem in 22] (cf. also Theorem 4.1 in [1]), this map sends $c_{*}(X) \otimes c_{*}(Y)$ to $c_{*}(X \times Y)$. Pushing forward to the ambient product of projective spaces, this says that $c_{*}\left(\mathbb{1}_{X \times Y}\right)$ is the image of $\left(f(h) \cap\left[\mathbb{P}^{m-1}\right]\right) \otimes\left(g(k) \cap\left[\mathbb{P}^{n-1}\right]\right)$; this is the statement.

Viewing $B \ell$ as the blow-up of $\mathbb{P}^{m+n-1}$ along the skew $\mathbb{P}^{m-1}$ and $\mathbb{P}^{n-1}$, let $E$ be the component of the exceptional divisor over $\mathbb{P}^{m-1}$, and $F$ the component over $\mathbb{P}^{n-1}$. Denote by $H$ the hyperplane class in $\mathbb{P}^{m+n-1}$, as well as its pull-back to $B \ell$.

The classes $H, h, k, E, F$ in $B \ell$ are not independent:

Lemma 6.4. $h=H-F$, and $k=H-E$. 
Proof. The projection $\mathbb{P}^{m+n-1}-\rightarrow \mathbb{P}^{m-1}$ is resolved by the blow-up $\widetilde{\mathbb{P}}^{m+n-1}$ of $\mathbb{P}^{m+n-1}$ along $\mathbb{P}^{n-1}$. It is clear that (the pull-back of) $h$ agrees with $H-F$ in this blow-up, where $F$ denotes the exceptional divisor over $\mathbb{P}^{n-1}$. This relation pulls back to the same relation in $B \ell$, which may be realized as the pull-back of $\widetilde{\mathbb{P}}^{m+n-1}$ along the inverse image of $\mathbb{P}^{m-1}$.

This proves the first relation. The second relation is obtained similarly.

By Lemma 6.3 and 6.4 the pull-back of $c_{*}\left(\mathbb{1}_{X \times Y}\right)$ to $B \ell$ is given by

$$
f(H-F) g(H-E) \cap[B \ell] \quad .
$$

The CSM class of $\rho^{-1}(X \times Y)$ may be obtained from this by applying a result of Shoji Yokura. For this, we note that $B \ell$ is smooth over $\mathbb{P}^{m-1} \times \mathbb{P}^{n-1}$, and more precisely $B \ell$ may be realized as the projectivization of $\mathcal{O}(-h) \oplus \mathcal{O}(-k)$. With this choice, the pull-back of $\mathcal{O}(H)$ agrees with the tautological bundle $\mathcal{O}(1)$ on $B \ell \cong \mathbb{P}(\mathcal{O}(-h) \oplus \mathcal{O}(-k))$.

\section{Lemma 6.5.}

$$
c_{*}\left(\mathbb{1}_{\rho^{-1}(X \times Y)}\right)=(1+F) f(H-F)(1+E) g(H-E) \cap[B \ell] .
$$

Proof. Write $\mathcal{E}=\mathcal{O}(-h) \oplus \mathcal{O}(-k)$, so $B \ell \cong \mathbb{P}(\mathcal{E})$. By Theorem 2.2 in [29], CSM classes behave like ordinary Chern classes through smooth morphisms: thus,

$$
c_{*}\left(\mathbb{1}_{\rho^{-1}(X \times Y)}\right)=c\left(T_{B \ell \mid\left(\mathbb{P}^{m-1} \times \mathbb{P}^{n-1}\right)}\right) \cap \rho^{*}\left(c_{*}\left(\mathbb{1}_{X \times Y}\right)\right) .
$$

The pull-back $\rho^{*}\left(c_{*}\left(\mathbb{1}_{X \times Y}\right)\right)=f(H-F) g(H-E) \cap[B \ell]$ was computed above. The relative tangent bundle $T_{B \ell \mid\left(\mathbb{P}^{m-1} \times \mathbb{P}^{n-1}\right)}$ is computed by means of the Euler exact sequence (cf. [15], B.5.8)

$$
0 \longrightarrow \mathcal{O} \longrightarrow \rho^{*} \mathcal{E} \otimes \mathcal{O}(H) \longrightarrow T_{B \ell \mid\left(\mathbb{P}^{m-1} \times \mathbb{P}^{n-1}\right)} \longrightarrow 0
$$

and gives $($ as $\mathcal{E}=\mathcal{O}(-h) \oplus \mathcal{O}(-k))$

$$
c\left(T_{B \ell \mid\left(\mathbb{P}^{m-1} \times \mathbb{P}^{n-1}\right)}\right)=c\left(\rho^{*} \mathcal{E} \otimes \mathcal{O}(H)\right)=(1-h+H)(1-k+H) .
$$

The statement follows from this and Lemma 6.4.

Example 6.6. For $X=\mathbb{P}^{m-1}, Y=\mathbb{P}^{n-1}$, we have $\rho^{-1}(X \times Y)=B \ell$, and $f(h)=$ $(1+h)^{m}-h^{m}, g(k)=(1+k)^{n}-k^{n}$. Noting that $h^{m}=0, k^{n}=0$, formula (6.2) gives

$$
c(T B \ell) \cap[B \ell]=(1+F)(1+H-F)^{m}(1+E)(1+H-E)^{n} \cap[B \ell] .
$$

This may also be obtained by two applications of Lemma 1.3 in [2], since $\mathbb{P}^{m-1}$ and $\mathbb{P}^{n-1}$ are disjoint complete intersections in $\mathbb{P}^{m+n-1}$.

These preliminary considerations yield the following statement.

Lemma 6.7. Let $\pi: B \ell \rightarrow \mathbb{P}^{m+n-1}$ be the blow-up along two disjoint centers $\mathbb{P}^{m-1}$, $\mathbb{P}^{n-1}$; let $E$, resp. $F$ be the exceptional divisors over these two centers; and let $H$ denote the hyperplane class in $\mathbb{P}^{m+n-1}$, as well as its pull-back to $B \ell$. For $X \subseteq \mathbb{P}^{m-1}, Y \subseteq \mathbb{P}^{n-1}$ nonempty subvarieties, let $f(H)$, resp. $g(H)$ be polynomial expressions of degrees $<m$, resp. $<n$ in $H$, such that

$$
c_{*}\left(\mathbb{1}_{X}\right)=f(H) \cap\left[\mathbb{P}^{m-1}\right] \quad, \quad c_{*}\left(\mathbb{1}_{Y}\right)=g(H) \cap\left[\mathbb{P}^{n-1}\right]
$$

as classes in $\mathbb{P}^{m+n-1}$. Finally, let $J(X, Y) \hookrightarrow \mathbb{P}^{m+n-1}$ be the join of $X$ and $Y$ in $\mathbb{P}^{m+n-1}$. Then

$$
\begin{aligned}
c_{*}\left(\mathbb{1}_{J(X, Y)}\right)=\pi_{*}((1+F) & (1+E) f(H-F) g(H-E) \cap[B \ell]) \\
& -(\chi(Y)-1) f(H) \cap\left[\mathbb{P}^{m-1}\right]-(\chi(X)-1) f(H) \cap\left[\mathbb{P}^{n-1}\right] .
\end{aligned}
$$

In this statement, $\chi(X)$ and $\chi(Y)$ denote the Euler characteristics of $X$ and $Y$, respectively. 
Proof. Realize the join $J(X, Y)$ as the image of $\rho^{-1}(X \times Y)$ in $\mathbb{P}^{m+n-1}$. Denote by $\bar{\pi}: \rho^{-1}(X \times Y) \rightarrow J(X, Y)$ the restriction of $\pi$. Then $\bar{\pi}$ is proper and birational, and contracts

$$
\begin{array}{ll}
E \cap \rho^{-1}(X \times Y) \quad \text { to } \quad X \subseteq \mathbb{P}^{m-1} \\
F \cap \rho^{-1}(X \times Y) \text { to } \quad Y \subseteq \mathbb{P}^{n-1} .
\end{array}
$$

Now, $E \cap \rho^{-1}(X \times Y) \cong X \times Y$, and the contraction corresponds to the projection $X \times Y \rightarrow X$. Similarly, the contraction $F \cap \rho^{-1}(X \times Y)$ to $Y$ corresponds to the projection $X \times Y \rightarrow Y$. Therefore, the fibers of $\bar{\pi}$ may be described as follows:

$$
\begin{aligned}
p \notin X \cup Y & \Longrightarrow \bar{\pi}^{-1}(p)=\text { a point } \\
p \in X & \Longrightarrow \bar{\pi}^{-1}(p) \cong Y \\
p \in Y & \Longrightarrow \bar{\pi}^{-1}(p) \cong X
\end{aligned}
$$

In terms of constructible functions, this says

$$
\begin{aligned}
\bar{\pi}_{*}\left(\mathbb{1}_{\rho^{-1}(X \times Y)}\right) & =\mathbb{1}_{J(X, Y) \backslash(X \cup Y)}+\chi(Y) \mathbb{1}_{X}+\chi(X) \mathbb{1}_{Y} \\
& =\mathbb{1}_{J(X, Y)}+(\chi(Y)-1) \mathbb{1}_{X}+(\chi(X)-1) \mathbb{1}_{Y} .
\end{aligned}
$$

By the functoriality property of Chern-Schwartz-MacPherson's classes, it follows that

$$
\bar{\pi}_{*} c_{*}\left(\mathbb{1}_{\rho^{-1}(X \times Y)}\right)=c_{*}(J(X, Y))+(\chi(Y)-1) c_{*}(X)+(\chi(X)-1) c_{*}(Y) .
$$

The statement follows immediately from this, together with Lemma 6.5

The challenge now is to evaluate the push-forward

$$
\pi_{*}((1+F)(1+E) f(H-F) g(H-E) \cap[B \ell]) .
$$

Since $f$ and $g$ are polynomials, this is a sum of terms

$$
\pi_{*}\left((1+F)(1+E)(H-F)^{i}(H-E)^{j} \cap[B \ell]\right) .
$$

This push-forward can be executed in two steps, since $\pi$ may be viewed as a composition $\pi=\pi_{2} \circ \pi_{1}$ of the blow-up $\pi_{1}$ of $\mathbb{P}^{m+n-1}$ along $\mathbb{P}^{m-1}$, followed by the blow-up $\pi_{2}$ of the resulting variety along (a locus isomorphic to) $\mathbb{P}^{n-1}$. Both steps match the following template:

Lemma 6.8. Let $p: \widetilde{V} \rightarrow V$ be the blow-up of a scheme $V$ along a subscheme $W$ of codimension $r$. Assume $W$ has class $H^{r}$, where $H$ is a divisor class in $V$. Denote by the same letter $H$ the pull-back of this divisor class to $\widetilde{V}$, and let $D$ be the exceptional divisor. Then

$$
p_{*}\left((H-D)^{j}\right)=\left\{\begin{array}{rl}
H^{j} & 0 \leq j<r \\
0 & j \geq r
\end{array} \quad, \quad p_{*}\left(D(H-D)^{j}\right)=\left\{\begin{array}{rl}
H^{r} & j=r-1 \\
0 & j \neq r-1
\end{array} .\right.\right.
$$

Proof. By the birational invariance of Segre classes (Proposition 4.2(a) in [15]),

$$
p_{*}\left(\frac{D}{1+D} \cap[\widetilde{V}]\right)=s(W, V)=\frac{H^{r}}{(1+H)^{r}} \cap[V] \quad,
$$

and hence

$$
p_{*}\left(\frac{1}{1+D} \cap[\widetilde{V}]\right)=\left(1-\frac{H^{r}}{(1+H)^{r}}\right) \cap[V] .
$$

Introducing a bookkeeping variable $v$, we have

$$
p_{*}\left(\frac{1}{1+v D} \cap[\widetilde{V}]\right)=\left(1-\frac{(v H)^{r}}{(1+v H)^{r}}\right) \cap[V] \quad:
$$

indeed, multiplying $D$ by $v$ on the left has the effect of multiplying every term of codimension $j$ by $v^{j}$, and this is the same effect obtained by multiplying $H$ by $v$ on the right. 
By the projection formula, $v$ may be replaced by any expression in $H$ on the right and by its pull-back on the left, still yielding a correct identity. Apply this observation to

$$
\sum_{j \geq 0}(H-D)^{j}=\frac{1}{1+D-H}=\frac{\frac{1}{1-H}}{1+\frac{D}{1-H}}
$$

with $v=\frac{1}{1-H}$ :

$$
\begin{aligned}
p_{*}\left(\sum_{j \geq 0}(H-D)^{j} \cap[\widetilde{V}]\right) & =\frac{1}{1-H} \cdot p_{*}\left(\frac{1}{1+\frac{1}{1-H} D} \cap[\widetilde{V}]\right) \\
& =\frac{1}{1-H} \cdot\left(1-\frac{\left(\frac{1}{1-H} H\right)^{r}}{\left(1+\frac{1}{1-H} H\right)^{r}}\right) \cap[V] \\
& =\frac{1}{1-H} \cdot\left(1-H^{r}\right) \cap[V] \\
& =\left(1+H+\cdots+H^{r-1}\right) \cap[V] .
\end{aligned}
$$

This establishes the first formula. The second formula follows immediately from this, by observing that

$$
D(H-D)^{j}=H(H-D)^{j}-(H-D)^{j+1} .
$$

Returning to our analysis of intersections in $B \ell$, Lemma 6.8 gives

\section{Lemma 6.9.}

$$
\begin{gathered}
\pi_{*}\left((H-F)^{i}(H-E)^{j} \cap[B \ell]\right)=\left\{\begin{array}{cl}
H^{i+j} \cap\left[\mathbb{P}^{m+n-1}\right] & \text { if } 0 \leq i<m \text { and } 0 \leq j<n \\
0 & \text { otherwise }
\end{array}\right. \\
\pi_{*}\left(E(H-F)^{i}(H-E)^{j} \cap[B \ell]\right)=\left\{\begin{array}{cl}
H^{i+n} \cap\left[\mathbb{P}^{m+n-1}\right] & \text { if } 0 \leq i<m \text { and } j=n-1 \\
0 & \text { otherwise }
\end{array}\right. \\
\pi_{*}\left(F(H-F)^{i}(H-E)^{j} \cap[B \ell]\right)=\left\{\begin{array}{cl}
H^{j+m} \cap\left[\mathbb{P}^{m+n-1}\right] & \text { if } 0 \leq j<n \text { and } i=m-1 \\
0 & \text { otherwise }
\end{array}\right. \\
\pi_{*}\left(E F(H-F)^{i}(H-E)^{j} \cap[B \ell]\right)=0 .
\end{gathered}
$$

Proof. The last formula follows from the fact that $E F=0$ (the two exceptional divisors are disjoint). The others are each obtained by applying Lemma 6.8 twice. For example, note that

$$
\pi_{*}\left((H-F)^{i}(H-E)^{j} \cap[B \ell]\right)=\pi_{1 *}\left((H-E)^{j} \cdot \pi_{2 *}\left((H-F)^{i} \cap[B \ell]\right)\right)
$$

by the projection formula, since $H, E$ are pull-backs from the first blow-up. Hence, Lemma 6.8 evaluates this class to

$$
\pi_{1 *}\left((H-E)^{j} \cdot H^{i}\right)
$$

if $0 \leq i<m\left(m=\right.$ the codimension of $\left.\mathbb{P}^{n-1}\right)$ and 0 otherwise; and another application of Lemma 6.8 evaluates this to $H^{i+j}$ if both $0 \leq i<m$ and $0 \leq j<n$, and 0 otherwise. The remaining two formulas are handled similarly.

We are finally ready to prove Theorem 3.13 
Proof of Theorem 3.13. We have to evaluate

$$
\pi_{*}((1+F)(1+E) f(H-F) g(H-E) \cap[B \ell]) .
$$

Let $f(x)=\sum_{i=0}^{m-1} a_{i} x^{j}$ and $g(x)=\sum_{j=0}^{n-1} b_{j} x^{i}$. Then

$$
\begin{gathered}
\pi_{*}((1+F)(1+E) f(H-F) g(H-E) \cap[B \ell]) \\
=\pi_{*}(f(H-F) g(H-F)+E f(H-F) g(H-E)+F f(H-F) g(H-E)) \\
=\sum_{i=0}^{m-1} \sum_{j=0}^{n-1} a_{i} b_{j} \pi_{*}\left((H-F)^{i}(H-E)^{j} \cap[B \ell]\right) \\
+\sum_{i=0}^{m-1} \sum_{j=0}^{n-1} a_{i} b_{j} \pi_{*}\left(E(H-F)^{i}(H-E)^{j} \cap[B \ell]\right) \\
+\sum_{i=0}^{m-1} \sum_{j=0}^{n-1} a_{i} b_{j} \pi_{*}\left(F(H-F)^{i}(H-E)^{j} \cap[B \ell]\right) \\
=\sum_{i=0}^{m-1} \sum_{j=0}^{n-1} a_{i} b_{j} H^{i+j}+\sum_{i=0}^{m-1} a_{i} b_{n-1} H^{i+n}+\sum_{j=0}^{n-1} a_{m-1} b_{j} H^{j+m} \\
=f(H) g(H)+\chi(Y) f(H) H^{n}+\chi(X) g(H) H^{m},
\end{gathered}
$$

using Lemma 6.9, and the fact that $\chi(X)=\int c_{*}(X)=a_{m-1}, \chi(Y)=\int c_{*}(Y)=b_{n-1}$. By Lemma 6.7 then,

$$
\begin{aligned}
c_{*}\left(\mathbb{1}_{J(X, Y)}\right)= & \left(f(H) g(H)+\chi(Y) f(H) H^{n}+\chi(X) g(H) H^{m}\right) \cap\left[\mathbb{P}^{m+n-1}\right] \\
& \quad-(\chi(Y)-1) f(H) \cap\left[\mathbb{P}^{m-1}\right]-(\chi(X)-1) f(H) \cap\left[\mathbb{P}^{n-1}\right] \\
= & \left(f(H) g(H)+f(H) H^{n}+g(H) H^{m}\right) \cap\left[\mathbb{P}^{m+n-1}\right] \\
= & \left(\left(f(H)+H^{m}\right)\left(g(H)+H^{n}\right)-H^{m+n}\right) \cap\left[\mathbb{P}^{m+n-1}\right] .
\end{aligned}
$$

This establishes formula (6.1), and concludes the proof of Theorem 3.13

\section{REFERENCES}

[1] P. Aluffi, Classes de Chern des variétés singulières, revisitées. C. R. Math. Acad. Sci. Paris, 342(6):405-410, 2006.

[2] P. Aluffi, Chern classes of blow-ups. arXiv:0809.2425

[3] P. Aluffi, M. Marcolli, Feynman motives of banana graphs. preprint arXiv:hep-th/0807.1690. To appear in Communications in Number Theory and Physics.

[4] V.I. Arnold, S.M. Gusein-Zade, A.N. Varchenko, Singularities of differentiable maps, Vol.II, Birkhäuser, 1988.

[5] J. Bjorken, S. Drell, Relativistic Quantum Mechanics, McGraw-Hill, 1964, and Relativistic Quantum Fields, McGraw-Hill, 1965.

[6] S. Bloch, Motives associated to graphs, Japan J. Math., Vol.2 (2007) 165-196.

[7] S. Bloch, Motives associated to sums of graphs, preprint arXiv:0810.1313

[8] S. Bloch, E. Esnault, D. Kreimer, On motives associated to graph polynomials, Commun. Math. Phys., Vol.267 (2006) 181-225.

[9] S. Bloch, D. Kreimer, Mixed Hodge structures and renormalization in physics, arXiv:0804.4399

[10] A. Connes, D. Kreimer, Renormalization in quantum field theory and the Riemann-Hilbert problem I. The Hopf algebra structure of graphs and the main theorem, Comm. Math. Phys., Vol.210 (2000) 249-273.

[11] A. Connes, D. Kreimer, Renormalization in quantum field theory and the Riemann-Hilbert problem. II. The $\beta$-function, diffeomorphisms and the renormalization group. Comm. Math. Phys. 216 (2001), no. 1, 215-241. 
[12] A. Connes, M. Marcolli, Noncommutative Geometry, Quantum Fields, and Motives, Colloquium Publications, Vol.55, American Mathematical Society, 2008.

[13] A. Connes, M. Marcolli, Quantum fields and motives, Journal of Geometry and Physics, Volume $56,(2005)$ N.1, 55-85.

[14] K. Ebrahimi-Fard, L. Guo, D. Kreimer, Spitzer's identity and the algebraic Birkhoff decomposition in $p Q F T$. J. Phys. A 37 (2004), no. 45, 11037-11052.

[15] W. Fulton. Intersection theory. Springer-Verlag, Berlin, 1984.

[16] L. Guo, Baxter algebras and the umbral calculus. in "Special issue in honor of Dominique Foata's 65th birthday (Philadelphia, PA, 2000)". Adv. in Appl. Math. 27 (2001), no. 2-3, 405-426.

[17] H. Gillet, C. Soulé, Descent, motives and K-theory, J. Reine angew. Math. 478 (1996) 127-176.

[18] C. Itzykson, J.B. Zuber, Quantum Field Theory, Dover Publications, 2006.

[19] S.A. Joni, G.C. Rota, Coalgebras and bialgebras in combinatorics, Stud. Appl. Math. 61 (1979) N.2, 93-139.

[20] M. Kapranov, The elliptic curve in the S-duality theory and Eisenstein series for Kac-Moody groups, arXiv:math.AG/0001005

[21] M. Kontsevich, D. Zagier, Periods, in "Mathematics unlimited - 2001 and beyond", pp.771-808, Springer, 2001

[22] M. Kwieciński, Formule du produit pour les classes caractéristiques de Chern-SchwartzMacPherson et homologie d'intersection. C. R. Acad. Sci. Paris Sér. I Math., 314(8):625-628, 1992.

[23] M. Larsen, V. Lunts, Motivic measures and stable birational geometry, Moscow Math. Journal, Vol.3 (2003) N.1, 85-95.

[24] R.D. MacPherson, Chern classes for singular algebraic varieties. Ann. of Math. (2) 100 (1974), 423-432.

[25] M. Marcolli, Motivic renormalization and singularities, arXiv:0804.4824

[26] M. Marcolli, A. Rej, Supermanifolds from Feynman graphs, Journal of Physics A, Vol.41 (2008) 315402 (21pp). arXiv:0806.1681

[27] G.C. Rota, Hopf algebra methods in combinatorics, in "Problèmes combinatoires et théorie des graphes" (Colloq. Internat. CNRS, Univ. Orsay, 1976) pp.363-365, Colloq. Internat. CNRS, 260, CNRS Paris, 1978.

[28] M.H. Schwartz, Classes caractéristiques définies par une stratification d'une variété analytique complexe, C. R. Acad. Sci. Paris, Vol.260 (1965) 3535-3537.

[29] S. Yokura. On a Verdier-type Riemann-Roch for Chern-Schwartz-MacPherson class. Topology Appl., 94(1-3):315-327, 1999. Special issue in memory of B. J. Ball.

Department of Mathematics, Caltech, Pasadena, CA 91125, USA

E-mail address: aluffi@caltech.edu

E-mail address: matilde@caltech.edu

Department of Mathematics, Florida State University, Tallahassee, FL 32306, USA

E-mail address: aluffi@math.fsu.edu

E-mail address: marcolli@math.fsu.edu

Max-Planck Institut für Mathematik, Vivatsgasse 7, Bonn, D 53111, Germany

E-mail address: aluffi@mpim-bonn.mpg.de

E-mail address: marcolli@mpim-bonn.mpg.de 